\title{
NUCLEAR INFRARED SPECTRAL ENERGY DISTRIBUTION OF TYPE II ACTIVE GALACTIC NUCLEI
}

\author{
Liza Videla ${ }^{1}$, Paulina Lira ${ }^{1}$, Heather Andrews ${ }^{2}$, Almudena Alonso-Herrero ${ }^{3}$, \\ DAVID M. AlexANDER ${ }^{4}$, AND MARTIN WARD ${ }^{4}$ \\ ${ }^{1}$ Departamento de Astronomía, Universidad de Chile, Chile \\ ${ }^{2}$ Leiden Observatory, Leiden University, NL-2300 RA Leiden, The Netherlands \\ ${ }^{3}$ Instituto de Física de Catabria, CSIC-UC, E-39005 Santander, Spain \\ ${ }^{4}$ Department of Physics, Durham University, South Road, Durham DH1 3LE, UK \\ Received 2012 February 20; accepted 2012 June 9; published 2013 February 5
}

\begin{abstract}
We present near- and mid-IR observations of a sample of Seyfert II galaxies drawn from the $12 \mu \mathrm{m}$ Galaxy sample. The sample was observed in the $J, H, K, L, M$ and $N$ bands. Galaxy surface brightness profiles are modeled using nuclear, bulge, bar (when necessary), and disk components. To check the reliability of our findings, the procedure was tested using Spitzer observations of M 31. Nuclear spectral energy distributions (SEDs) are determined for 34 objects, and optical spectra are presented for 38, including analysis of their stellar populations using the STARLIGHT spectral synthesis code. Emission line diagnostic diagrams are used to discriminate between genuine active galactic nuclei (AGNs) and $\mathrm{H}$ II nuclei. Combining our observations with those found in the literature, we have a total of 40 SEDs. It is found that about $40 \%$ of the SEDs are characterized by an upturn in the near-IR, which we have quantified as a NIR slope $\alpha<1$ for an SED characterized as $\lambda f_{\lambda} \propto \lambda^{\alpha}$. The three objects with an $\mathrm{H}$ II nucleus and two Seyfert nuclei with strong contamination from a circumnuclear also show an upturn. For genuine AGNs, this component could be explained as emission from the accretion disk, a jet, or from a very hot dust component leaking from the central region through a clumpy obscuring structure. The presence of a very compact nuclear starburst as the origin for this NIR excess emission is not favored by our spectroscopic data for these objects.
\end{abstract}

Key words: galaxies: active - galaxies: nuclei - galaxies: Seyfert

Online-only material: color figures, figure set, machine-readable table

\section{INTRODUCTION}

\subsection{Unified Model}

The presence of well-collimated radio jets in radio loud active galactic nuclei (AGNs) and the expectation of an axis of symmetry defined by the geometrically thin accretion disk argue for strong anisotropies in the central engines of AGNs. This implies that the appearance of an AGN will depend on the observer's position relative to the system's axis. Following this reasoning, and to explain a wealth of observational evidence, the Unified Model postulates that Type I and Type II AGNs are the same kind of object, but that they appear distinct because of the difference in angle between the central engine's axis and our line of sight.

Support for this model has been particularly strong in the case of Seyfert galaxies. Optical spectra of Seyfert I objects show broad emission line regions (BLR); those of Seyfert II objects do not. Explaining this as an orientation effect, with obscuration of the central source, goes back to at least Osterbrock (1978), who suggested that both types are physically the same, but that in Seyfert II galaxies the BLR is blocked from our view, probably by dust in a toroidal structure surrounding the central source. Below we summarize some of the work that followed and supported this thesis.

Evidence for obscuration of Type II objects comes from the excess absorption in X-ray, UV, and optical wavelengths, when observations are compared with Type I nuclei (Lawrence \& Elvis 1982; Mass-Hesse et al. 1993; Turner et al. 1997; Malkan et al. 1998; Risaliti et al. 1999). Recently, Shi et al. (2006) have shown a clear correlation between the column densities derived from X-ray data and the strength of the $9.7 \mu \mathrm{m}$ silicate feature: Type II systems with large hydrogen columns show large absorption features, while objects classified as Type I often show the feature in emission.

In those AGNs that are radio-loud sources, the radio structure appears to be aligned with the torus axis (Wilson \& Tsvetanov 1994; Nagar \& Wilson 1999; Barbosa et al. 2009). This anisotropy is also observed in the cone-like structures of the narrow line region, where the torus collimates ionizing radiation from the central source (Pogge 1988a, 1988b; Falcke et al. 1998).

In the IR, the detection of broad components from hydrogen recombination lines such as $\mathrm{Pa} \beta$ and $\mathrm{Br} \gamma$ in objects optically classified as Seyfert II galaxies is consistent with extinction along a line-of-sight that grazes the torus surface, sufficient to suppress the BLR emission at optical wavelengths, but optically thin in the infrared (Ruiz et al. 1994; Veilleux et al. 1997, 1999).

More direct support for unification comes from the Hubble Space Telescope (HST), which has imaged an extended nuclear structure of $\sim 90$ pc in NGC 4261 (Jaffe et al. 1993), while interferometry observations are starting to shed light on the inner obscuring torus (Wittkowski et al. 1998, 2004; Weinberger et al. 1999; Swain et al. 2003; Meisenheimer et al. 2007; Tristram et al. 2007, 2009; Beckert et al. 2008; Kishimoto et al. 2009, 2011; Raban et al. 2009).

There is also evidence for structure in the obscuring material. Changes in broad line profiles (Penston \& Perez 1984) and inferred column densities imply the presence of clumpy structures, leading to variable obscuration of the central engine as they move across the line of sight (Risaliti et al. 2011, 2009; Puccetti et al. 2007; Elvis et al. 2004; although the column density variations are more likely to correspond to obscuration by BLR clouds than the torus). 
Finally, the most famous, and arguably most convincing evidence in support of the Unified Model, comes from optical spectropolarimetry of Type II objects. Antonucci \& Miller (1985) found scattered emission from the BLR in polarized flux from NGC 1068, strongly supporting the idea of unification by orientation, and so implying the existence of an obscuring structure. Their work was extended by (Young et al. 1995, 1996; Moran et al. 2000).

\subsection{Spectral Energy Distribution}

The spectral energy distribution (SED) is an important tool for studying the central engine in AGNs. In most luminous and intermediate-luminosity AGNs it can be characterized by two bumps-one peaking in the UV and the other in the mid-IR - and an inflection point inbetween, at $\sim 1.5 \mu \mathrm{m}$ (Elvis et al. 1994). The UV feature (the "big blue bump") is normally identified with emission from the central accretion disk, while the IR flux is assumed to originate in the dusty obscuring structure, the torus. The latter has a broad SED, with a peak somewhere between 5-30 $\mu \mathrm{m}$, decreasing slowly toward the far-IR. The emission mechanism is believed to be thermal reprocessing of central, optical-UV emission by dust at a wide range of temperatures - the highest $(\sim 1000-1500 \mathrm{~K})$ corresponding to dust closest to the central engine.

Obtaining the SED of the torus in AGNs is difficult for a number of reasons.

First, the SED inflection point matches the peak in starlight emission from the host galaxy. In intermediate-luminosity Seyfert galaxies the host galaxy contribution is often comparable to the emission from the nucleus, flattening the optical-IR region of the SED and lowering the relative strength of the UV and IR bumps. To study the nuclear SED, galactic starlight must be removed.

Second, until recently, only small size IR arrays have been available, making it impossible to image the complete host galaxy for nearby sources, compromising the modeling of the surface brightness distribution.

Third, observations at $\lambda \gtrsim 10 \mu \mathrm{m}$ can only be achieved from space, which are characterized by lower angular resolution.

Fourth, and finally, observations obtained at different epochs can be affected by variability. Fortunately, the torus region is large, damping most variations of the central source, so this is not expected to be significant.

The number of compiled AGN SEDs in the literature increases constantly, but many of them incorporate data gathered by different groups using different instruments; this often implies data of different resolutions, analyzed in different ways. To properly study the emission from the dusty torus in AGNs, and to accurately infer physical and geometrical characteristics, this paper describes a more homogenous dataset. We have constructed nuclear IR SEDs of a sample of 48 Type II Seyfert galaxies using near- and mid-IR high-resolution ground-based images (described here), and modeled them in clumpy using the approach of Nenkova et al. (2002, 2008a, 2008b; described in a companion paper, Lira et al. 2013, hereafter Paper II). The main contribution of this work is the number of objects we study, and the detailed treatment of the nuclear SED construction.

In Section 2 we present the sample, observations, and data reduction, including the optical spectroscopy and the stellar population analysis. Section 3 describes, in detail, the path from IR images to the final nuclear IR SEDs of our sample, including the analysis of M 31 to validate our methodology. Section 4 discusses the results and possible interpretations. A summary is presented in Section 5.

\section{OBSERVATIONS}

The sample used in this work contains all Seyfert II galaxies from the Extended $12 \mu \mathrm{m}$ Galaxy Sample (Rush et al. 1993) that are located in the southern hemisphere. The 48 objects are described in Table 1.

The $12 \mu \mathrm{m}$ Galaxy sample has several advantages over other catalogs. Most importantly, it is selected in the mid-infrared (MIR), giving a more representative population of nearby $(z \leqslant 0.07)$ AGNs. In comparison, optically selected samples can miss obscured systems and are more susceptible to the effects of host galaxy reddening (e.g., Huchra \& Burg 1992; Maiolino et al. 1995). This is important because a wide range of nuclear obscuration (hydrogen columns of $\sim 10^{22}-10^{25} \mathrm{~cm}^{-2}$ ) can provide more general tests of nuclear emission models. In addition, the $12 \mu \mathrm{m}$ sample includes elliptical, lenticular and spiral galaxies (which helps avoid systematic errors in the decomposition process of the surface brightness profiles) and a wide range of galaxy inclinations (which allows the importance of galactic, as well as nuclear, absorption to be assessed).

However, the sample may be biased toward Seyfert II sources with strong star formation (Buchanan et al. 2006) - the stellar IR emission can compensate for a weak AGN component, increasing the likelihood of inclusion in a flux limited sample. This might explain the prevalence of star formation in Seyfert II galaxies when compared with Seyfert I nuclei (e.g., Maiolino et al. 1995).

Our analysis of the central region of galaxies is built on extensive studies of the $12 \mu \mathrm{m}$ Galaxy sample at many wavelengths, from radio to X-rays (Spinoglio et al. 1995, 2002; Rush et al. 1996a; Hunt \& Malkan 1999, Hunt et al. 1999; Bassani et al. 1999; Thean et al. 2000, 2001; Imanishi 2003; Imanishi \& Alonso-Herrero 2004; Gorjian et al. 2004; Strong et al. 2004; Buchanan et al. 2006; Tommasin et al. 2008, 2010; Wu et al. 2009; Gallimore et al. 2010; Baum et al. 2010), including spectropolarimetric observations (Tran 2001).

The initial classification of objects in the sample, by Rush et al. (1993), was based on existing catalogs of active galaxies (Veron-Cetty \& Veron 1991; Hewitt \& Burbidge 1991). Since then, as better optical spectra became available, some objects have been re-classified. To verify the classifications, and to study stellar populations, we obtained high-quality optical spectroscopy for 38 objects. The analysis of these data is presented next.

\subsection{Optical Spectroscopy and Analysis}

Long-slit optical spectroscopy was obtained with the R-C Spectrograph on the Blanco $4 \mathrm{~m}$ telescope at CTIO, in 2007 August and 2008 February. We used the KPGL3-1 grating and a slit width of $1^{\prime \prime}$. The slit was positioned at the parallactic angle to minimize light losses. In both runs the seeing varied between $\sim 0.8$ and $\sim 1.2$ arcsec. The February 2008 run was photometric, while flux calibration is not necessary to characterize the nebular and stellar components of the nuclear spectra.

The data were reduced using IRAF ${ }^{5}$ software. Standard reduction procedures (bias subtraction, flat-fielding, slit illumination correction) were followed. Extraction of the galactic spectra

\footnotetext{
5 IRAF is distributed by the National Optical Astronomy Observatory, which is operated by the Association of Universities for Research in Astronomy, Inc., under cooperative agreement with the National Science Foundation.
} 
Table 1

Observed Sample of 48 Seyfert 2 Galaxies, Indicating the Detector Used in Each Infrared Observation

\begin{tabular}{|c|c|c|c|c|c|c|c|c|}
\hline $\begin{array}{l}\text { Galaxy } \\
\text { (1) }\end{array}$ & $\begin{array}{c}\text { Hubble Type } \\
\text { (2) }\end{array}$ & $\begin{array}{l}\text { Redshift } \\
\text { (3) }\end{array}$ & $\begin{array}{l}\text { Inc } \\
(4)\end{array}$ & $\begin{array}{c}\text { R.A. } \\
(5)\end{array}$ & $\begin{array}{l}\text { Decl. } \\
(6)\end{array}$ & $\begin{array}{c}J H K \text { bands } \\
\text { (7) }\end{array}$ & $\begin{array}{c}L M \text { bands } \\
\text { (8) }\end{array}$ & $\begin{array}{c}N \text { band } \\
\text { (9) }\end{array}$ \\
\hline NGC 34, MRK 938 & $2 \mathrm{Sc}$ & 0.0198 & 0.14 & 001106.55 & -120626.32 & ISA-2 & ISA71-B & \\
\hline F $00198-7926$ & 2 Pec & 0.0728 & 0.31 & 002152.90 & -791008.00 & ISA-2 & ISA71-B & $\ldots$ \\
\hline F $00521-7054$ & E-S0 & 0.0689 & 0.22 & 005356.15 & -703804.16 & ISA-2 & ISA71-B & $\ldots$ \\
\hline ESO 541-IG12 & Mult & 0.0564 & 0.29 & 010217.55 & -194008.67 & ISA-2 & ISA71-B & G04 \\
\hline NGC 424, TOL0109 & $(\mathrm{R}) \mathrm{SB}(\mathrm{r}) 0 / \mathrm{a}$ & 0.0117 & lost & 011127.51 & -380501.08 & ISA-2 & ISA71-B & $\ldots$ \\
\hline F 01475-0740 & E-S0 & 0.0177 & 0.17 & 015002.70 & -072548.48 & ISA-2 & ISA71-B & $\ldots$ \\
\hline NGC 1068 & ? (R)SA(rs)b & 0.0038 & 0.07 & 024240.71 & -000047.81 & AH03 & AH03 & AH03 \\
\hline NGC 1097 & $\left(\mathrm{R}^{\prime} \_1\right) \mathrm{SB}\left(\mathrm{r}^{\prime} 1\right) \mathrm{b}$ & 0.0042 & lost & 024618.99 & -301628.68 & ISA-2 & ISA71-B & $\ldots$ \\
\hline NGC 1125 & $\mathrm{SAB} 0$ & 0.0109 & $\ldots$ & 025140.27 & -163903.69 & $\ldots$ & ISA71-B & $\ldots$ \\
\hline NGC 1144 & S0 pec & 0.0288 & 0.08 & 025510.85 & -001047.20 & ISA-2 & ISA71-B & NED \\
\hline MCG -2-8-39, F 02581-1136 & $\mathrm{SAB}(\mathrm{rs}) \mathrm{a}$ & 0.0299 & 0.64 & 030030.69 & -112454.06 & ISA-2 & ISA71-B & NED \\
\hline NGC 1194 & $\mathrm{SA} 0+$ & 0.0136 & 0.46 & 030349.11 & -010613.39 & ISA-2 & $\ldots$ & G04 \\
\hline NGC 1241 & $\mathrm{SB}(\mathrm{rs}) \mathrm{b}$ & 0.0135 & lost & 031114.90 & -085520.89 & ISA-2 & $\ldots$ & $\ldots$ \\
\hline NGC 1320, MRK 607 & 乙 Sa sp & 0.0094 & 0.69 & 032448.72 & -030231.99 & ISA-2 & $\ldots$ & G04 \\
\hline NGC 1386 & $\mathrm{SB}(\mathrm{s}) 0+$ & 0.0029 & lost & 033646.40 & -360002.00 & ISA-2 & $\ldots$ & $\ldots$ \\
\hline F $03362-1642$ & Pec & 0.0369 & 0.68 & 033834.54 & -163215.84 & ISA-2 & $\ldots$ & G04 \\
\hline F 04385-0828 & S0 & 0.0151 & 0.54 & 044054.96 & -082222.22 & ISA-2 & $\ldots$ & G04 \\
\hline NGC 1667 & $\mathrm{SAB}(\mathrm{r}) \mathrm{c}$ & 0.0152 & 0.31 & 044837.14 & -061911.87 & ISA-2 & $\ldots$ & GEMI \\
\hline ESO 33-G2 & SB0 & 0.0186 & 0.34 & 045558.99 & -753228.06 & ISA-2 & $\ldots$ & $\ldots$ \\
\hline F 05189-2524 & Pec & 0.0426 & 0.06 & 052101.47 & -252145.38 & ISA-2 & $\ldots$ & GEMI \\
\hline ESO 253-G3 & Sa tidal & 0.0425 & $2 n u c$ & 052518.29 & -460019.60 & ISA-2 & $\ldots$ & GEMI \\
\hline $\mathrm{MCG}+0-29-23$ & $\mathrm{SAB}(\mathrm{s}) \mathrm{b}$ & 0.0248 & 0.07 & 112112.26 & -025903.45 & SofI(S) & ISA70-B & G04 \\
\hline NGC 3660, MRK 1291 & $\mathrm{SB}(\mathrm{r}) \mathrm{bc}$ & 0.0123 & 0.14 & 112332.24 & -083930.21 & SofI(S) & ISA70-B & GEMI \\
\hline NGC 4388 & $\mathrm{SA}(\mathrm{s}) \mathrm{b} \mathrm{sp}$ & 0.0084 & 0.64 & 122546.75 & +123943.51 & AH03 & AH03 & G04 \\
\hline NGC 4501, M 88 & $\mathrm{SA}(\mathrm{rs}) \mathrm{b}$ & 0.0076 & 0.47 & 123159.16 & +142513.60 & SofI(S) & ISA70-B & GEMI-G04 \\
\hline TOL $1238-364$, IC 3639 & 2 SB(rs)bc & 0.0109 & 0.12 & 124052.88 & -364521.52 & SofI(S) & ISA70-B & GEMI \\
\hline NGC 4941 & R)SAB(r)ab & 0.0037 & 0.50 & 130413.06 & -05335.79 & SofI(S) & ISA70-B & G04 \\
\hline NGC 4968 & ? (R')SAB0 & 0.0099 & 0.34 & 130705.89 & -234039.38 & AH03 & AH03 & G04 \\
\hline MCG -3-34-64, F 13197-1672 & SB? & 0.0165 & 0.25 & 132224.46 & -164342.90 & SofI(S) & ISA70-B & G04 \\
\hline NGC 5135 & ¿ SB(1)ab & 0.0137 & 0.01 & 132543.97 & -295002.26 & SofI(S) & ISA70-B & G04 \\
\hline MRK 463 & Merger & 0.0503 & $2 n u c$ & 135602.87 & +182219.48 & SofI(S) & ISA70-B & G04 \\
\hline NGC 5506 & Sa pec sp & 0.0062 & 0.52 & 141314.86 & -031226.94 & AH03 & AH03 & G04 \\
\hline NGC 5953, MRK 9031 & SAa pec & 0.0065 & 0.37 & 153433.70 & +151149.50 & SofI(S) & ISA70-B & GEMI-G04 \\
\hline NGC 5995, MCG -2-40-4 & $\mathrm{S}(\mathrm{B}) \mathrm{c}$ & 0.0252 & 0.19 & 154824.96 & -134527.94 & SofI(S) & ISA70-B & G04 \\
\hline F $15480-0344$ & 2 S0 & 0.0303 & 0.11 & 155041.51 & -035318.34 & SofI(S) & ISA71-B & G04 \\
\hline F $19254-7245$ & Merger & 0.0617 & $2 n и с$ & 193121.40 & -723917.96 & SofI(V) & ISA71-B & GEMI \\
\hline NGC 6810, F 19393-5846 & SA(s)ab:sp & 0.0068 & 0.50 & 194334.16 & -583920.54 & SofI(V) & ISA71-B & GEMI \\
\hline NGC 6890 & $\left.\mathrm{R}^{\prime}\right) \mathrm{SA}(\mathrm{r}:) \mathrm{ab}$ & 0.0081 & 0.15 & 201818.07 & -444823.36 & SofI(V) & ISA71-B & GEMI \\
\hline IC 5063 & $\mathrm{SA}(\mathrm{s}) 0+$ & 0.0114 & 0.2 & 205202.01 & -570409.12 & SofI(V) & ISA71-B & GEMI \\
\hline MRK 897, UGC 11680 & Compact & 0.0264 & 0.21 & 210743.70 & +035219.00 & SofI(V) & ISA71-B & GEMI \\
\hline NGC 7130, IC 5135 & 2 Sa pec & 0.0162 & 0.01 & 214819.49 & -345705.98 & SofI(V) & ISA71-B & GEMI \\
\hline NGC 7172 & Sa pec sp & 0.0087 & 0.25 & 220201.68 & -315218.12 & AH03 & AH03 & G04 \\
\hline MCG $-3-58-7$, F 22469-1932 & $\left(\mathrm{R}^{\prime}\right) \mathrm{SAB}(\mathrm{s}) 0 / \mathrm{a}$ & 0.0317 & 0.13 & 224937.15 & -191626.39 & SofI(V) & ISA71-B & G04 \\
\hline NGC 7496 & $\left(\mathrm{R}^{\prime}\right) \mathrm{SB}(\mathrm{rs}) \mathrm{bc}$ & 0.005 & 0.11 & 230947.26 & -432539.76 & SofI(V) & ISA71-B & $\ldots$ \\
\hline NGC 7582 & $\left(\mathrm{R}^{\prime} \_1\right) \mathrm{SB}(\mathrm{s}) \mathrm{ab}$ & 0.0053 & 0.69 & 231823.50 & -422213.98 & SofI(SV) & ISA71-B & $\ldots$ \\
\hline NGC 7590 & $\mathrm{~S}(\mathrm{r} ?) \mathrm{bc}$ & 0.0053 & 0.64 & 231854.60 & -421421.00 & SofI(SV) & ISA71-B & $\ldots$ \\
\hline NGC 7674, MRK 533 & 2 SA(r)bc pec & 0.0289 & 0.04 & 232756.74 & +084644.52 & AH03 & AH03 & G04 \\
\hline CGCG 381-051, F 23461+0157 & $\mathrm{SBc}$ & 0.0307 & 0.10 & 234841.64 & +021424.02 & SofI(V) & ISA71-B & G04 \\
\hline
\end{tabular}

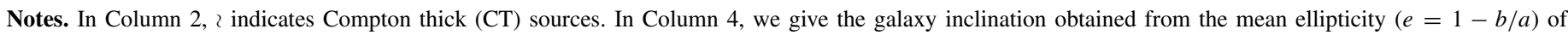

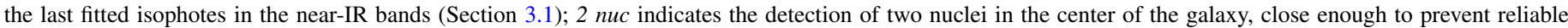

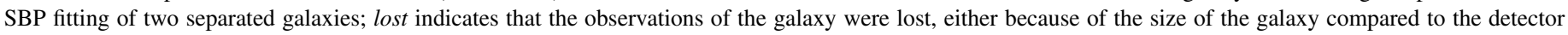

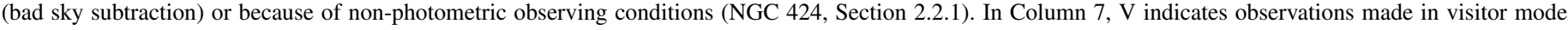

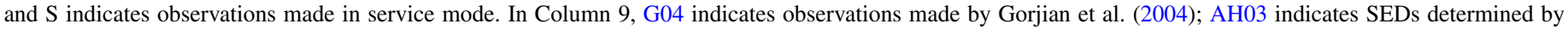
Alonso-Herrero et al. (2003); NED indicates values obtained from the NASA/IPAC Extragalactic Database ${ }^{\mathrm{a}}$.

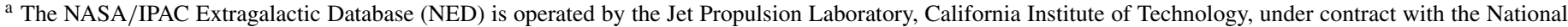
Aeronautics and Space Administration.

used a window of width $\sim 2$ '.2. Wavelength calibration used comparison copper-neon and copper-argon lamps. The spectra were corrected for the foreground extinction tabulated by Schlegel et al. (1998) using the empirical extinction function of Cardelli et al. (1989).
We determined the stellar component of the spectra using the STARLIGHT spectral synthesis code developed by Cid Fernandes et al. (2004, 2005). A total of 45 different stellar population templates were used, covering an age range of $\sim 10^{6}-10^{10}$ years and a metallicity range of $0.2-2.5 \times Z_{\odot}$. The 
best-fit spectrum was found by minimizing $\chi^{2}$ after masking all emission lines.

Following Cid Fernandes et al. (2004, 2005), we grouped output from the population synthesis into three age groups: a Young component (Y: $t<10^{8} \mathrm{yr}$ ), an intermediate component (I: $10^{8}<t<10^{9} \mathrm{yr}$ ), and an old component (O: $t>2.5 \times 10^{9} \mathrm{yr}$ ). The results are presented in Table 2.

The best-fit stellar models were subtracted from the observed data to give pure emission line spectra. This reveals weak emission lines present in targets with significant intermediateage stellar populations. Table 2 contains the measured emission line flux ratios.

Diagnostic diagrams were constructed using emission line fluxes and are shown in Figure 1. The diagrams allow us to distinguish different ionizing mechanisms of the emission nebula using line ratios that are sensitive to changes in the spectral shape of the ionizing radiation (Baldwin et al. 1981; Veilleux \& Osterbrock 1987; Kewley et al. 2001). In this way we can test the identification of the sources as Seyfert nuclei.

We will discuss possible contaminants in Figure 1 by type. First, we argue that there is little evidence for LINERs in our sample.

In panel (a), there are no data points in the LINER region. The border between the HII and LINER regions is defined to include all star-forming models (it is calculated from the highest possible line ratios), but the $[\mathrm{N}$ II $] \lambda 6584 / \mathrm{H} \alpha$ ratio for star-forming regions and LINERs can overlap (Kewley et al. 2001), so LINERs can be located to the left of the border. Also, theoretical models of shock excitation give line ratios in the LINER region only when no precursor is observed (Allen et al. 2008). For both these reasons panel (a) does not discriminate these two classes of object well.

In panel (b), variations in density can introduce uncertainties in the $\left[\mathrm{S}_{\mathrm{II}}\right] \lambda \lambda 6717,6730 / \mathrm{H} \alpha$ ratio, so objects should not be classified as a LINER based purely on this diagnostic (Kewley et al. 2001).

Given the above, and the single LINER candidate in panel (c) which gives the most reliable test, none of our sources can be firmly classified as a LINER nucleus.

Second, four sources systematically appear in the HII section of the diagrams, consistent with the classification of Gallimore et al. (2010). These are NGC 5953 (but see next), MCG +0-29-23, NGC 6810, and MRK 897. Also, NGC 34 appears systematically close to the starburst region.

NGC 5953 was classified as an AGN by Rafanelli et al. (1990) after making a careful identification of the nuclear source, which can be confused with a bright star-forming region located $3^{\prime \prime}$ to the west. Unfortunately, we centered our slit on the star-formation knot and the slit orientation did not include the nucleus, so our optical spectrum is of the wrong object. The IR images, however, are clearly dominated by the nuclear region-the star-forming region can also be seen, but is much less prominent. The determined SED, however, suggests that contamination of this circumnuclear starburst or a compact nuclear starburst is present (Section 4).

One of the most reliable signatures of an active nucleus is luminous, hard X-ray emission. We checked the literature for X-ray data on MCG +0-29-23, NGC 6810, and MRK 897, but found little support for classification as Seyferts. Strickland (2007) presents the discovery of a starburst driven wind in NGC 6810 from XMM-Newton observations, and explains that the claim of a hard $L_{\mathrm{x}} \sim 10^{42} \mathrm{erg} \mathrm{s}^{-1}$ source in this galaxy was due to the misidentification of a HEAO1 source. Upper
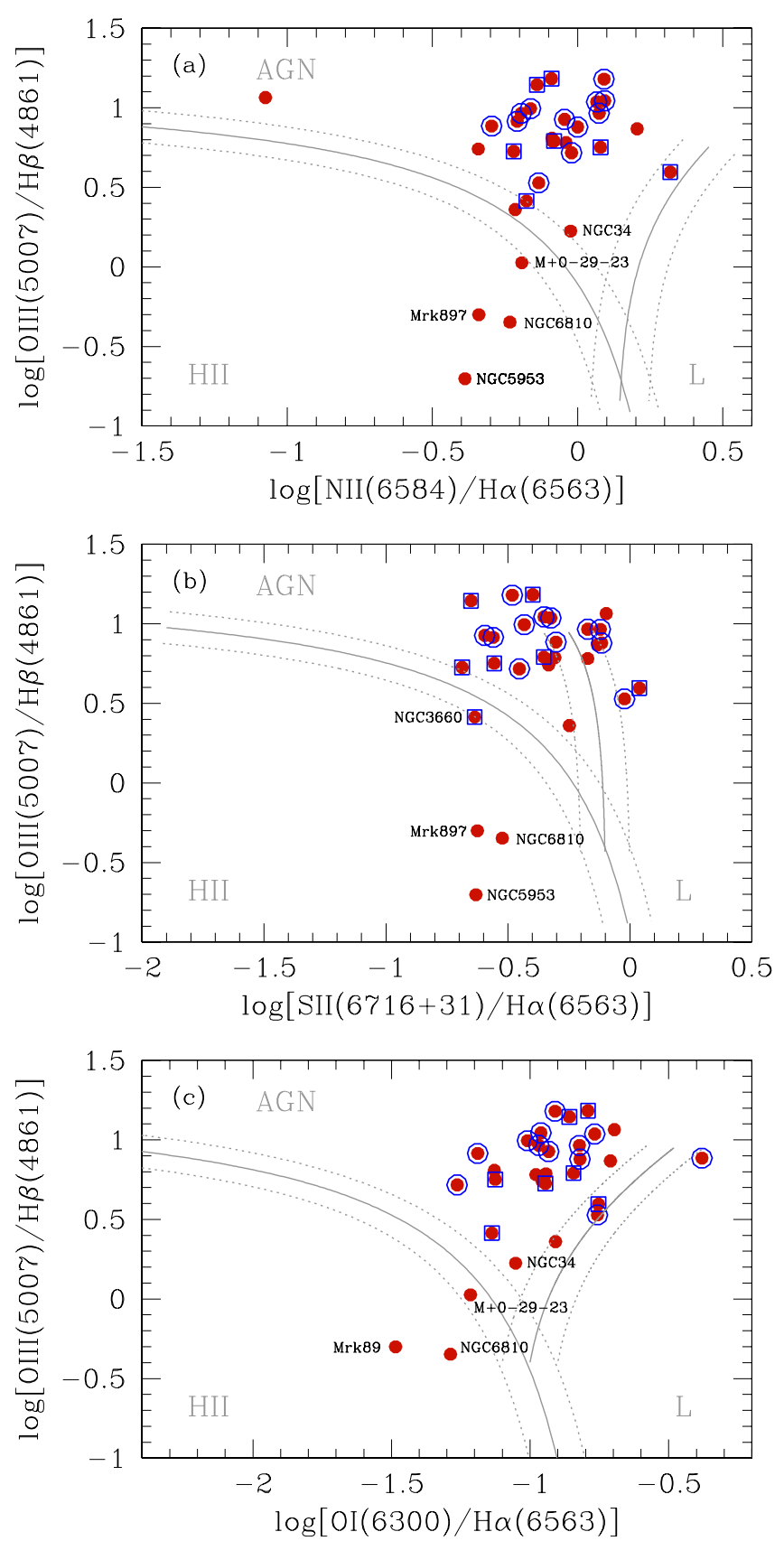

Figure 1. Diagnostic diagrams for emission line spectra. The theoretical divisions between the star-forming, AGN, and LINER classes, as parameterized by Kewley et al. (2001), are shown with thick gray lines in panels (a), (b), and (c). Dotted lines indicate \pm 0.1 dex. Nuclei that fall in the $\mathrm{H}$ II region are labeled. Also, AGN nuclei are shown with an extra circle or square if they have a NIR slope grater or smaller than 1 , respectively (see Section 4).

(A color version of this figure is available in the online journal.)

limits of $L_{\mathrm{x}}<1 \times 10^{42}$ and $6 \times 10^{41} \mathrm{erg} \mathrm{s}^{-1}$ in the soft band were reported by Rush et al. (1996a) for MRK 897 and MCG +0-29-23.

Given the above, NGC 5953 a seems to harbor a genuine Seyfert II nucleus, but MCG +0-29-23, NGC 6810, and MRK 897 do not. Contamination by a starburst component in NGC 34 and NGC 5953 seems likely. We will present data for all the objects in this work, but exclude the three canonical H II nuclei from analysis in Paper II. 
Table 2

Population Synthesis Analysis and Measured Emission Line Fluxes and Flux Ratios

\begin{tabular}{|c|c|c|c|c|c|c|c|c|c|c|}
\hline Name & $\begin{array}{c}\mathrm{Y}(\%) \\
(2)\end{array}$ & $\begin{array}{c}\mathrm{I}(\%) \\
(3)\end{array}$ & $\begin{array}{c}\mathrm{O}(\%) \\
(4)\end{array}$ & $\begin{array}{c}f(4861) \\
(5)\end{array}$ & $\begin{array}{c}f(5007) \\
(6)\end{array}$ & $\begin{array}{c}(5007 / 4861) \\
(7)\end{array}$ & $\begin{array}{c}(6583 / 6563) \\
(8)\end{array}$ & $\begin{array}{c}(6300 / 6563) \\
(9)\end{array}$ & $\begin{array}{c}(6716 / 6563) \\
(10)\end{array}$ & $\begin{array}{c}(6563 / 4861) \\
(11)\end{array}$ \\
\hline NGC 34 & 46.8 & 34.6 & 18.6 & 0.9 & 1.6: & 0.2 : & -0.02 & -1.1 & $\ldots$ & 13.7 \\
\hline F 00198-7926 & 0.0 & 0.0 & 100.0 & 0.5 & 3.9 & $\ldots$ & $\ldots$ & $\ldots$ & $\ldots$ & $\ldots$ \\
\hline F 00521-7054 & 0.0 & 10.6 & 89.4 & 0.8 & 6.8 & 0.9 & -0.05 & -0.9 & -0.6 & 8.3: \\
\hline ESO 541-IG12 & 53.6 & 0.0 & 46.4 & 0.7 & 5.8 & 0.9 & -0.2 & -1.2 & -0.6 & 6.1: \\
\hline NGC 424 & 23.9 & 0.0 & 76.1 & 10.0 & 54.5 & $\ldots$ & $\ldots$ & $\ldots$ & $\ldots$ & $\ldots$ \\
\hline F 01475-0740 & 53.6 & 0.0 & 46.4 & 1.4 & 7.5 & 0.7 & -0.2 & -0.9 & -0.7 & 7.1 \\
\hline NGC 1068 & $\ldots$ & $\ldots$ & $\ldots$ & $\ldots$ & $\ldots$ & $\ldots$ & $\ldots$ & $\ldots$ & $\ldots$ & $\ldots$ \\
\hline NGC 1097 & $\ldots$ & $\ldots$ & $\ldots$ & $\ldots$ & $\ldots$ & $\ldots$ & $\ldots$ & $\ldots$ & $\ldots$ & $\ldots$ \\
\hline NGC 1125 & 50.3 & 3.9 & 45.8 & 2.4 & 14.7 & 0.8 & -0.09 & -0.9 & -0.3 & 4.8 \\
\hline NGC 1144 & 0.0 & 0.0 & 100.0 & 0.6 & 6.4 & $\ldots$ & $\ldots$ & $\ldots$ & $\ldots$ & $\ldots$ \\
\hline $\mathrm{MCG}-2-8-39$ & 21.8 & 0.0 & 78.2 & 1.8 & 26.8 & 1.2 & -0.09 & -0.8 & -0.4 & 4.2 \\
\hline NGC 1194 & 26.7 & 0.0 & 73.3 & 0.3 & 2.6 & 0.9 & -0.3 & -0.4 & -0.3 & 6.6 \\
\hline NGC 1241 & $\ldots$ & $\ldots$ & $\ldots$ & $\ldots$ & $\ldots$ & $\ldots$ & $\ldots$ & $\ldots$ & $\ldots$ & $\ldots$ \\
\hline NGC 1320 & 4.80 & 15.9 & 79.3 & 2.6 & 25.5 & 1.0 & -0.2 & -1.0 & -0.4 & 3.8 \\
\hline NGC 1386 & $\ldots$ & $\ldots$ & $\ldots$ & $\ldots$ & $\ldots$ & $\ldots$ & $\ldots$ & $\ldots$ & $\ldots$ & $\ldots$ \\
\hline F 03362-1642 & 51.6 & 0.0 & 48.4 & 0.7 & 4.5 & 0.8 & -0.09 & -1.1 & $\ldots$ & 5.3 \\
\hline F 04385-0828 & 45.8 & 0.0 & 54.2 & 0.5 & 1.8 & 0.5 & -0.1 & -0.8 & -0.02 & 4.0: \\
\hline NGC 1667 & 25.2 & 0.0 & 74.8 & 1.1 & 7.9 & 0.9 & 0.2 & -0.7 & -0.1 & 5.7: \\
\hline ESO 33-G2 & 32.3 & 1.2 & 66.5 & 1.3 & 12.4 & 1.0 & 0.07 & -1.0 & -0.2 & 5.0: \\
\hline F 05189-2524 & 1.9 & 26.7 & 71.4 & 1.9 & 11.0 & $\ldots$ & $\ldots$ & $\ldots$ & $\ldots$ & $\ldots$ \\
\hline ESO 253-G3 & 75.6 & 0.0 & 24.4 & 2.7 & 15.1 & 0.7 & -0.3 & -1.0 & -0.3 & 3.6: \\
\hline $\mathrm{MCG}+0-29-23$ & 28.2 & 10.7 & 61.1 & 0.8 & 0.9: & 0.02: & -0.2 & -1.2 & $\ldots$ & 8.1 \\
\hline NGC 3660 & 3.3 & 0.0 & 96.7 & 1.3 & 3.5 & 0.4 & -0.2 & -1.1 & -0.6 & 3.3: \\
\hline NGC 4388 & 35.2 & 0.0 & 64.8 & 4.9 & 45.2 & 1.0 & -0.2 & -0.8 & -0.1 & 4.0 \\
\hline NGC 4501 & 7.8 & 0.0 & 92.2 & 0.9 & 3.6: & 0.6: & 0.3 & -0.8 & 0.04 & 3.7: \\
\hline TOL 1238-364 & 26.2 & 20.5 & 53.3 & 8.1 & 49.8 & 0.8 & -0.08 & -0.8 & -0.4 & 4.0 \\
\hline NGC 4941 & 0.0 & 0.0 & 100.0 & 2.8 & 31.9 & 1.0 & -1.1 & -0.7 & -0.1 & 4.6 \\
\hline NGC 4968 & 28.7 & 6.2 & 65.1 & 2.4 & 26.0 & 1.0 & 0.07 & -0.8 & -0.3 & 4.3 \\
\hline MCG -3-34-64 & 0.0 & 22.0 & 78.0 & 16.3 & $\ldots$ & $\ldots$ & $\ldots$ & $\ldots$ & $\ldots$ & $\ldots$ \\
\hline NGC 5135 & 53.9 & 16.9 & 29.2 & 4.5 & 23.3 & 0.7 & -0.02 & -1.3 & -0.5 & 5.6 \\
\hline MRK $463 \mathrm{a}^{\mathrm{a}}$ & 60.1 & 23.7 & 16.2 & 1.1 & 2.5 & 0.4 & -0.2 & -0.9 & -0.2 & 3.2 \\
\hline MRK 463ba & 63.6 & 19.1 & 17.3 & 9.2 & 67.0 & $\ldots$ & $\ldots$ & $\ldots$ & $\ldots$ & $\ldots$ \\
\hline NGC 5506 & 53.1 & 0.0 & 46.9 & 9.2 & 59.7 & 0.9 & 0.0 & -0.8 & -0.1 & 4.4 \\
\hline NGC 5953 & 66.6 & 12.2 & 21.2 & 8.0 & 0.3: & $-0.7:$ & -0.4 & $\ldots$ & -0.6 & 4.2 \\
\hline MCG $-2-40-4$ & 27.3 & 4.2 & 68.5 & 1.6 & 8.8 & $\ldots$ & $\ldots$ & $\ldots$ & $\ldots$ & $\ldots$ \\
\hline F $15480-0344$ & 11.2 & 13.8 & 75.0 & 1.7 & 65.0 & 1.1 & -0.1 & -0.9 & -0.7 & 4.6: \\
\hline F $19254-7245$ & 18.9 & 0.0 & 81.1 & 4.7 & 4.3 & $\ldots$ & $\ldots$ & $\ldots$ & $\ldots$ & $\ldots$ \\
\hline NGC 6810 & 44.2 & 2.1 & 53.7 & 0.6 & 1.1: & -0.3 : & -0.2 & -1.3 & -0.5 & 7.4: \\
\hline NGC 6890 & 19.2 & 6.2 & 74.5 & 2.5 & 15.0 & 1.2 & 0.09 & -0.9 & -0.5 & 5.0 \\
\hline IC 5063 & $\ldots$ & $\ldots$ & $\ldots$ & 1.0 & $\ldots$ & $\ldots$ & $\ldots$ & $\ldots$ & $\ldots$ & $\ldots$ \\
\hline MRK 897 & 34.3 & 38.2 & 27.5 & $\ldots$ & 1.5 & -0.3 & -0.3 & -1.5 & -0.6 & 4.3 \\
\hline NGC 7130 & 65.8 & 12.1 & 22.1 & 3.0 & 27.1 & 0.8 & 0.08 & -1.1 & -0.6 & 7.9 \\
\hline NGC 7172 & $\ldots$ & $\ldots$ & $\ldots$ & 4.8 & $\ldots$ & $\ldots$ & $\ldots$ & $\ldots$ & $\ldots$ & $\ldots$ \\
\hline MCG -3-58-7 & 33.6 & 27.8 & 38.7 & $\ldots$ & 18.6 & 1.0 & 0.09 & -1.0 & -0.4 & 4.7 \\
\hline NGC 7582 & $\ldots$ & $\ldots$ & $\ldots$ & 5.9 & $\ldots$ & $\ldots$ & $\ldots$ & $\ldots$ & $\ldots$ & $\ldots$ \\
\hline NGC 7590 & 23.0 & 8.0 & 69.0 & $\ldots$ & 3.3 & 0.8 & -0.04 & -1.0 & -0.2 & 5.9 \\
\hline NGC 7674 & $\ldots$ & $\ldots$ & $\ldots$ & 0.5 & $\ldots$ & $\ldots$ & $\ldots$ & $\ldots$ & $\ldots$ & $\ldots$ \\
\hline CGCG 381-051 & $\ldots$ & $\ldots$ & $\ldots$ & $\ldots$ & $\ldots$ & $\ldots$ & $\ldots$ & $\ldots$ & $\ldots$ & $\ldots$ \\
\hline
\end{tabular}

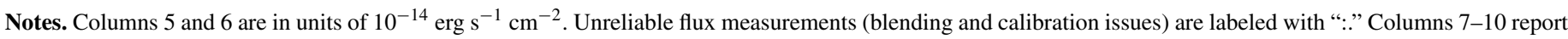
the logarithm of the flux ratios. Column 11 is the Balmer decrement.

a Spectra for both nuclei in MRK 463 were obtained.

\subsection{Imaging and Data Reduction}

The following galaxies in our sample have existing highquality SEDs in Alonso-Herrero et al. (2003): NGC 1068, NGC 4388, NGC 4968, NGC 5506, NGC 7172, and NGC 7674. The remaining 42 Type II Seyfert galaxies were observed in the following infrared bands: near-Infrared (NIR) $J(1.25 \mu \mathrm{m})$, $H(1.65 \mu \mathrm{m})$, and $K(2.2 \mu \mathrm{m})$ bands; and mid-IR (MIR) $L$ $(3.56 \mu \mathrm{m}), M(4.66 \mu \mathrm{m})$, and $N(10 \mu \mathrm{m})$ bands. Details of the observing runs are listed in Table 3; typical seeing conditions (for an $\mathrm{H}_{0}=70 \mathrm{~km} \mathrm{~s}^{-1} \mathrm{Mpc}^{-1}$ ) are summarized in Table 4 .
Table 3

Summary of Infrared Observing Runs

\begin{tabular}{lcclc}
\hline \hline Period & Telescope & Detector & Mode & Bands \\
\hline 2002B & ANTU (UT1) & ISAAC & Visitor & $J H K$ \\
2002B & ANTU (UT1) & ISAAC & Service & $L M$ \\
2003A & ANTU (UT1) & ISAAC & Service & $L M$ \\
2003B & NTT & SofI & Service & $J H K$ \\
2004A & Gemini S & T-ReCS & Service & $N$ \\
2004B & NTT & SofI & Visitor & $J H K$
\end{tabular}


Table 4

Summary of the Seeing Conditions

\begin{tabular}{lcccccc}
\hline \hline Band & $\begin{array}{c}\text { Mean } \\
\left({ }^{\prime \prime}\right)\end{array}$ & $\begin{array}{c}\sigma \\
\left({ }^{\prime \prime}\right)\end{array}$ & $\begin{array}{c}\text { Median } \\
\left({ }^{\prime \prime}\right)\end{array}$ & $\begin{array}{c}\text { Mean } \\
(\mathrm{pc})\end{array}$ & $\begin{array}{c}\sigma \\
(\mathrm{pc})\end{array}$ & $\begin{array}{c}\text { Median } \\
(\mathrm{pc})\end{array}$ \\
\hline$J$ & 0.7 & 0.1 & 0.7 & 325 & 317 & 224 \\
$H$ & 0.7 & 0.1 & 0.6 & 318 & 319 & 203 \\
$K$ & 0.6 & 0.1 & 0.6 & 296 & 283 & 208 \\
$L$ & 0.4 & 0.1 & 0.4 & 206 & 179 & 127 \\
$M$ & 0.4 & 0.1 & 0.4 & 227 & 178 & 162 \\
$N$ & 0.4 & 0.1 & 0.3 & 105 & 103 & 69 \\
\hline
\end{tabular}

\subsubsection{NIR Observations}

Observations in the near-IR bands followed the usual strategy for this wavelength regime: several images of short integration time (3-20 s) are obtained to avoid saturating the detector and to minimize fluctuations in sky brightness; the location of the object on the detector is changed for each image to subtract the sky efficiently and to avoid bad pixels.

The data were reduced, in part, with the XMOSAIC task from the IRAF XDIMSUM package. Darks were subtracted and flat fields applied. Sky images were calculated from a masked median of the object images or from the mean of sky-only images.

Sky images were subtracted and results were registered and shifted before co-adding. The standard deviation per pixel was calculated from an exposure map.

The observations with ISAAC in visiting mode were done under non-photometric conditions. Flux calibration was achieved using field stars found in the 2 MASS catalog ${ }^{6}$ as standard flux calibrators. This strategy did not work for NGC 424, which had no field stars in the galaxy frame, and those observations were lost.

\subsubsection{MIR Observations}

At these wavelengths the atmosphere is very bright, and temporarily and spatially variable; the telescope and surroundings also emit and reflect radiation. Thus observations were made using standard chopping and nodding techniques.

Mid-IR detectors are small, and the probability of finding a star in the same frame as the galaxy is slim, so each mid-IR galaxy observation was followed by observation of a star to determine the point-spread function (PSF). Known standards were used for flux calibration.

The ISAAC mid-IR observations were reduced with the ECLIPSE "jitter" pipeline. In a number of cases observing conditions degraded at the end of the integration, so some of the nodding series were discarded by hand.

The Gemini $N$-band data were reduced using the MIDIR task from the IRAF GEMINI package. When pipeline results were compared with a reduction by hand the images were very similar.

\section{CONSTRUCTING THE SEDs}

The SEDs contain nuclear magnitudes extracted from nearand mid-IR images. In Section 3.1, we describe how onedimensional (1D) brightness profiles are calculated from 2D images; the modeling of these profiles to separate the nuclear component is described in Section 3.2.

\footnotetext{
6 This publication makes use of data products from the Two Micron All Sky Survey, which is a joint project of the University of Massachusetts and the Infrared Processing and Analysis Center/California Institute of Technology, funded by the National Aeronautics and Space Administration and the National Science Foundation.
}

\subsection{Determining the Surface Brightness Profiles}

Deep IR images of a sample of Type II Seyfert galaxies were obtained. These images cover the entire galaxy, but only the nuclear emission is needed for the SED. To deconvolve the galaxy into its different components-bulge, disk, bar, and nucleus - we first determine the radial surface brightness profile (SBP) of the galaxy (Kotilainen et al. 1992; Zitelli et al. 1993; Alonso-Herrero et al. 1996; Prieto et al. 2001, 2002). The main advantage of this 1D approach is that smaller structures (spiral arms, HiI Regions, rings) are diluted relative to the more fundamental structures (bulge, disk, nucleus, and bar) and the quantity of data that must be fitted is reduced from $\sim 500 \times 500$ pixels to $\sim 200$ points.

The task ELLIPSE from the IRAF STSDAS package is used on the sky-subtracted images to obtain the SBPs. A manual masking removes contamination from different sources that might distort the isophote fitting, like foreground bright stars or other galaxies. ELLIPSE fits ellipses to the galaxy isophotes; these are described by the center coordinates, the position angle and the ellipticity. The center coordinates were held fixed while the other parameters were allowed to vary. A linear radial sampling was used, with steps of 0.5-1 pixel, depending on the size of the galaxy. In cases where the edge of the galaxy was too faint and the ellipse fitting process did not converge, the fitted isophotes observed in other images were adopted. In order to take into account the inclination of the galaxy, the SBP was corrected using the mean ellipticity of the last fitted isophotes.

\subsection{Modeling the Surface Brightness Profiles}

Most spiral galaxies can be described using a disk, bulge, optional bar, and, sometimes, an active nucleus. Historically, each component has been modeled with a specific analytical function. These are described below.

Nucleus. The nuclear point source is modeled using Dirac's Delta

$$
\int_{-a}^{a} \delta(t) d t=1 .
$$

Thus, the nuclear SBP is described by

$$
\Sigma_{n}(r)=\sigma_{n} \delta(r),
$$

where $\sigma_{n}$ is the nuclear surface brightness.

Disk. The disk is modeled using an exponential law

$$
\Sigma_{d}(r)=\sigma_{d} \exp \left\{-r / r_{d}\right\}
$$

where $\sigma_{d}$ is the surface brightness of the disk at $r=0$, $\sigma_{d}=\Sigma_{d}(r=0)$, and $r_{d}$ is the disk scale length.

Bulge. The bulge is modeled using a general Sérsic's law

$$
\Sigma_{B}(r)=\sigma_{B} \exp \left\{-b_{n} \cdot\left(r / r_{B}\right)^{1 / n}\right\},
$$

where $n$ is a free parameter and $b_{n}$ is defined by

$$
\Gamma(2 n)=2 \gamma\left(2 n, b_{n}\right)
$$

where $\Gamma$ is the (complete) gamma function, and $\gamma(2 n, x)$ is the incomplete gamma function defined by

$$
\gamma(2 n, x)=\int_{0}^{x} e^{-t} t^{2 n-1} d t .
$$


Common values of $b_{n}$ are $b_{1}=1.678$, corresponding to an exponential SBP, and $b_{4}=7.6692$, corresponding to a de Vaucouleur's profile. The most usual analytical expressions for $b_{n}$ are $b_{n}=1.9992 \cdot n-0.3271$ for $0.5<n<10$, and $b_{n}=2 n-1 / 3$ for $n \gtrsim 8$ (Graham 2001; Graham \& Driver 2005). In this work, we adopt the first approximation because the typical values of $n$ are between 0.5 and 4 in the IR bands (Balcells et al. 2003; Hunt et al. 2004; Grosbøl et al. 2004; Kormendy \& Kennicutt 2004).

Bar. Many galaxies in the sampled are classified as barred, and approximately two-thirds of all spiral galaxies classified as non-barred look barred in the IR (Kormendy \& Kennicutt (2004), and references therein). In order to model the bar, a second general Sérsic's law was included in the model, which introduced three new free parameters.

Final Model. The final model is the sum of the three or four components convolved with the image PSF in order to introduce the effect of the seeing:

$$
\begin{aligned}
\Sigma(r)= & {\left[\sigma_{n} \delta(r)+\sigma_{d} e^{-\frac{r}{r_{d}}}+\sigma_{B} e^{-b_{n_{B}}\left(\frac{r}{r_{B}}\right)^{1 / n_{B}}}\right.} \\
& \left.+\sigma_{b} e^{-b_{n_{b}}\left(\frac{r}{r_{b}}\right)^{1 / n_{b}}}\right] \otimes \operatorname{PSF}(r) .
\end{aligned}
$$

\subsubsection{The Point-spread Function}

To determine the correct parameters for the galaxy profile, we need a precise representation of the PSF describing the seeing and instrument response of each observation. For our NIR imaging, the PSF is very accurately determined from a star found in the galaxy frame, which guarantees the same distortion for galaxy and star. In the MIR imaging, the star is observed before and/or after the galaxy and, as the integration is quite long at these wavelengths, seeing conditions can change significantly. This means that the MIR stellar PSF may not accurately match the galaxy. In such cases, the bulge profile is taken from the NIR fit, but scaled to match the external part of the observed profiles, and the nuclear flux was obtained assuming $F_{\text {nuc }}=F_{\text {gal }}-F_{\text {bul }}$. See the Appendix for details.

The PSF radial profile was obtained using the same technique as the galaxy profile (Section 3.1) and then modeled with a Moffat function to allow for interpolation/extrapolation when fitting the galaxy profile. The Moffat function is similar to a Gaussian, but has more extended tails that better fit the effects of seeing, as shown in Figure 2. It is given by

$$
\begin{gathered}
\operatorname{Moff}(r)=\frac{\text { peak }}{\left(1+(r / \alpha)^{2}\right)^{\beta}} . \\
\text { 3.2.2. The Fitting Routine }
\end{gathered}
$$

\subsubsection{The Fitting Routine}

To measure the nuclear flux-i.e., to deconvolve the nuclear emission from that of the galaxy - the radial SBP was modeled using the expression described above (Equation (7)). The fitting routine uses the "Downhill Simplex Method in Multidimensions" first proposed by Nelder \& Mead (1965): the minimization process starts with an initial guess from the user and then takes a series of steps exploring the available parameter space of the problem until it achieves the termination criterion which is typically an insignificant variation between one step and the next.

As the algorithm does not guarantee that the minimum found is global, it was started from four or five extreme points of the parameter space: the first initial guess includes all the galactic components of the model with comparable contributions to the

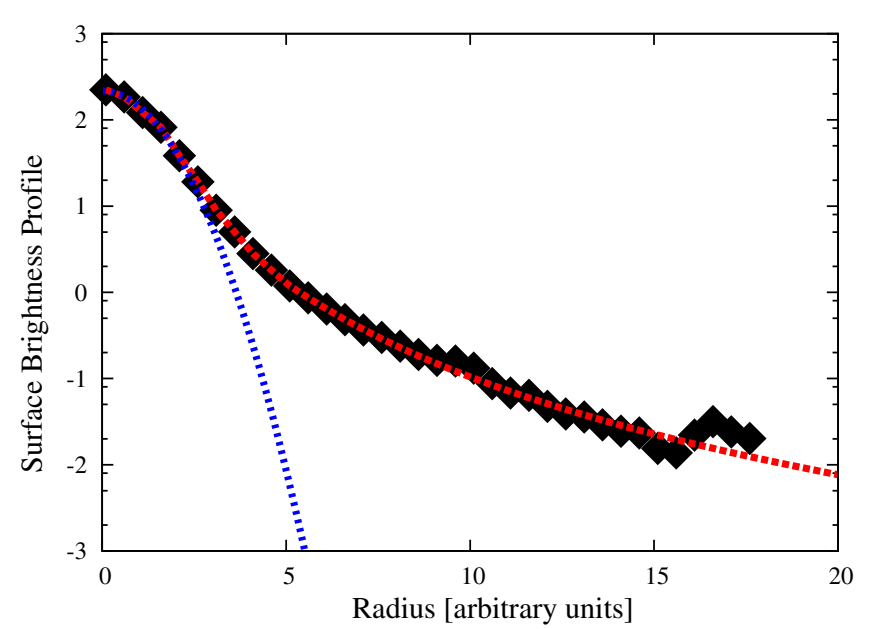

Figure 2. Example of the Moffat fit of a PSF star. The observed data are the black diamonds and the Moffat profile is represented by the red line. A Gaussian profile is shown for comparison (blue).

(A color version of this figure is available in the online journal.)

total flux; other initial points each zero one of the components. These first guesses are not completely blind, but instead are based on the morphology seen in the images. An iterative process that randomly perturbs the initial point is also included. Tests showed that 200 iterations were sufficient to reliably find the global minimum, which corresponds to the model that minimizes $\chi^{2}$; the percentage contributions of the galactic components are obtained from this model.

\subsubsection{Errors in the Fitted Components}

The flux of each fitted galactic component has two main sources of uncertainty: the first from the flux calibration (the zero points, obtained by the standard calibration procedure) and the second from the fitting process. The confidence limits for the fitted parameters, determined using the fitting routine described previously, are calculated using Monte Carlo simulations: the best-fit parameters are perturbed (one at a time) to construct many synthetic data sets. Each of these synthetic data sets has a particular $\chi_{\text {syn }}^{2}$ value and the first confidence level (corresponding to $1 \sigma$ error), which occurs $68.3 \%$ of the time, corresponds to the cases where $\Delta \chi^{2}=\chi_{\min }^{2}-\chi_{\text {syn }}^{2} \leqslant 1$. The final errors are calculated as the quadratic sum of the individual errors for the two sources of uncertainty.

\subsubsection{Synthetic Data}

Synthetic data were used to test the fitting routines. SBPs of different "kinds" of galaxies were constructed: including all components equally; all components with very small or very large contributions; omitting one or more components; with wrong PSFs; and with different sampling steps (for the galaxy and the PSF star).

The first test recovered the parameters of the synthetic SBP. The routine gives excellent estimates of the parameters for galaxies composed of three or four structural parameters with non-negligible contributions to the total emission. In cases when one or more components were not present, the results were also good, corresponding to observed examples like the non-barred galaxies, or a couple of cases where neither disk nor bar is observed. Including all the four components might give a better $\chi^{2}$ because there will be more free parameters, but if the image does not show a bar, for example, it is obvious that it should not be included in the fit. 

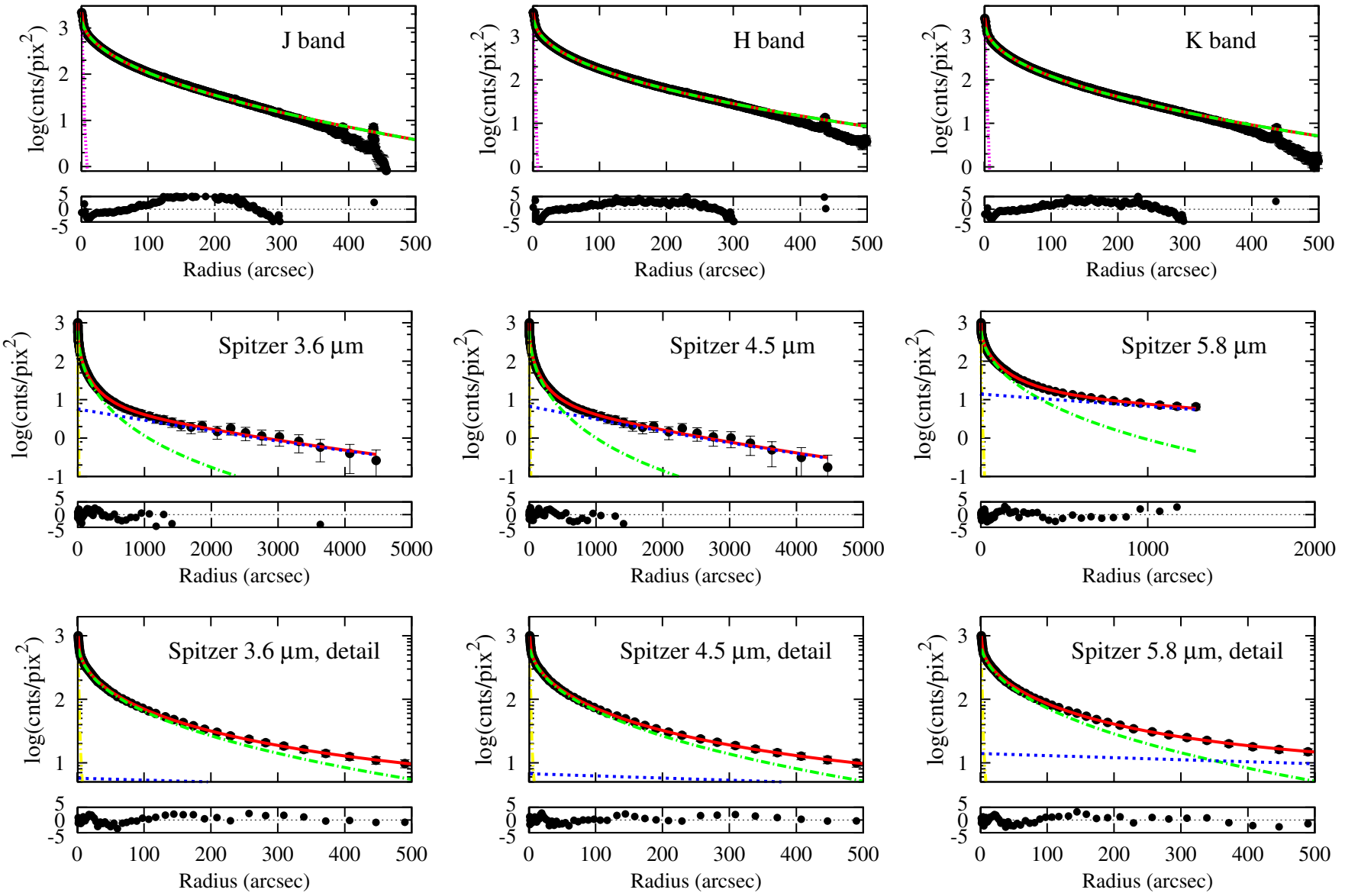

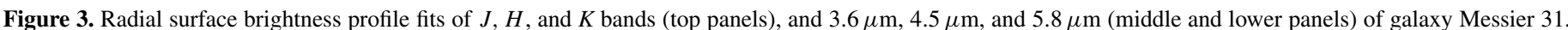

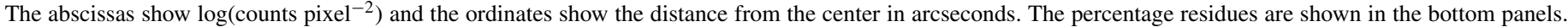

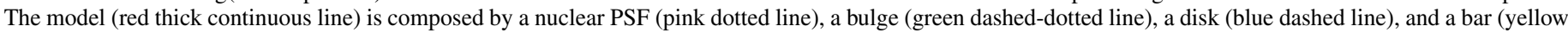

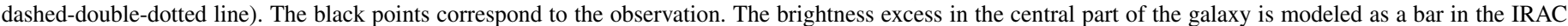
bands and as a nuclear component in the 2MASS bands. See the text for details.

(A color version of this figure is available in the online journal.)

Next, changes in the PSF were used to test the effects on the results (i.e., using a wrong PSF to describe the seeing conditions). We found that the results could be recovered (that is, at least two of the initial starting points could reach the actual solution) whenever the FWHM was narrower or broader by up to $50 \%$ of its real value. These tests apply especially to the midIR observations: as the integration time is long, the probability of a change in the seeing conditions between the observation of the PSF star and galaxy is greater.

The truncation of the galaxy profile severely affects the performance of the fitting routine. Fortunately, there are very few such cases in the observations, and then, typically, only one of the broadband images is affected, which means that results can be checked with the unaffected profiles and parameters constrained appropriately.

Finally, it is found that the sampling step is not particularly important, provided that the PSF is sampled by a finer discretization than the sampling of the galaxy.

\subsection{Messier 31}

To test the SBP fitting procedure, Messier 31, the Andromeda galaxy, was studied in the same way as the objects in our sample.

Images of M31 were taken by IRAC as part of the Spitzer General Observer program, ID 3126, in 2005 August. Barmby et al. (2006) measured the SBPs by fitting elliptical isophotes to the images using the ELLIPSE task. Profiles in the three longer wavelength bands (at $4.5,5.8$, and $8.0 \mu \mathrm{m}$ ) were measured using the ellipses fitted to the $3.6 \mu \mathrm{m}$ image, so that colors could be measured at the same spatial locations from all four bands. These profiles were kindly provided by P. Barmby (Barmby et al. 2006).

Images in the $J, H$, and $K$ bands were obtained from the 2MASS public catalog. The profiles were constructed using ELLIPSE. However, M31 is so close that the 2MASS images do not cover the full extent of the galaxy: while Spitzer-IRAC covers $\gtrsim 4000^{\prime \prime}$ in radius, the images of 2MASS cover only $\sim 500^{\prime \prime}$. This compromises the results for the disk component in the 2MASS data.

M31 is one of the nearest galaxies, and all galactic components can be detected in all the IR bands, which is not the case for the galaxies in our sample. The SBPs of M31 were fitted from $1.25 \mu \mathrm{m}$ to $5.8 \mu \mathrm{m}$ (Figure 3). The $8.0 \mu \mathrm{m} \mathrm{SBP}$ was not included because it is dominated by a $10 \mathrm{Kpc}$ star-forming ring and individual stellar features. The $5.8 \mu \mathrm{m}$ SBP was truncated at $\sim 1300^{\prime \prime}$ to avoid wiggles produced by the $10 \mathrm{Kpc}$ ring. The relevant fitted parameters are listed in Table 5.

It is interesting to note the bright excess in the central part of the galaxy profiles. This excess is modeled as a bar in the IRAC bands and as a nucleus in the 2MASS bands (due to the difference in pixel scale). Peng (2002) studied the central region of M31 in detail using the HST. He modeled the center of the galaxy using six components to account for three different 
Table 5

Values for the Relevant Fitted Parameters of M 31 Deconvolution Process

\begin{tabular}{lcccccc}
\hline \hline Component & $1.25 \mu \mathrm{m}$ & $1.65 \mu \mathrm{m}$ & $2.2 \mu \mathrm{m}$ & $3.6 \mu \mathrm{m}$ & $4.5 \mu \mathrm{m}$ & $5.8 \mu \mathrm{m}$ \\
\hline Bulge $r_{\text {eff }}$ & 185 & 228 & 207 & 301 & 259 & 209 \\
Bulge $n$ & 2.0 & 2.3 & 2.2 & 2.5 & 2.4 & 2.2 \\
Disk $r$ & $\ldots$ & $\ldots$ & $\ldots$ & 1165 & 991 & 996 \\
Bar $r_{\text {eff }}$ & $\ldots$ & $\ldots$ & $\ldots$ & 1.2 & 1.2 & 0.6 \\
Bar $n$ & $\ldots$ & $\ldots$ & $\ldots$ & 0.6 & 0.5 & 1.2 \\
\hline
\end{tabular}

Notes. The scale lengths are expressed in arcseconds. The bright excess in the central part of the galaxy profiles is modeled as a bar in the IRAC bands and as a nuclear component in the 2MASS bands (due to the differences in pixel scale). The disk is not included in the 2MASS fits because of the smaller image coverage. See the text for details.

peaks of emission in UV and optical images. However, the UV peak disappears in the near-IR images and the two $V$-band peaks diminish, so here we assume that the nucleus/bar fitted by the process is real, but the pixel scale does not allow a more detailed modeling.

As the disk could not be fitted in the 2MASS profiles, only the fitted radius of the bulge is compared: $700 \mathrm{pc}$ in the $J$ band, $862 \mathrm{pc}$ in the $H$ band, $787 \mathrm{pc}$ in the $K$ band, $1140 \mathrm{pc}$ at $3.6 \mu \mathrm{m}$, $983 \mathrm{pc}$ at $4.5 \mu \mathrm{m}$, and $795 \mathrm{pc}$ at $5.8 \mu \mathrm{m}$. The dispersion of these values about the mean is between $20 \%$ and $30 \%$ and they show no significant systematic change moving toward longer wavelengths.

The results corroborate some naïve expectations: unless there are strong color gradients within each of the structural components (the bulge, disk, and/or bar), the scale lengths should remain fairly constant for the galaxies in our sample, while the brightness scale factor should decrease from the NIR to the MIR as the stellar populations become dimmer. Also, since the bulge has older stars and is redder than the disk and the bar, it is expected to be the brightest component in the mid-IR.

Given these findings for M 31, we decided, when fitting the SBPs of the galaxies in our sample, to constrain the scale lengths in the MIR using the values fitted in the NIR, since the very dim stellar components underdetermine the fitting at these wavelengths. See the Appendix for details.

\section{GALAXY FITTING RESULTS}

The fitting of the SBPs for each of the galaxies in our sample was done assuming the model described in Section 3.2. Visual inspection of the images and the Hubble classification of each galaxy were used to check for coherent results.

Fitted parameters are generally consistent between observing bands. The following is a brief summary of the results, which are also presented in Table 6.

No disk or bar was detected in the $M$ band, while the bulge was detected in $7 / 28$ sources. In those objects the bulge contributed a mean brightness of $44 \%$ to the total flux. Only one bar was detected in the $L$ band with a contribution of $17 \%$. In the NIR bulge, disk and bar (if present) have similar contributions of $\sim 30 \%-50 \%$.

Out of 105 near-IR fits, 7 require a null nuclear contribution. The mean percentage contribution of each galactic component is summarized in Table 6 . A mean nuclear contribution of $\lesssim 10 \%$ is found in the NIR bands (JHK), while a strong increase can be seen in the MIR, going from $\sim 40 \%$ in the $M$ band to $\sim 100 \%$ in the $M$ and $N$ bands.

The percentage contribution of the nuclear component in the $J, K, L$, and $M$ bands is shown in Figure 4 as a function of

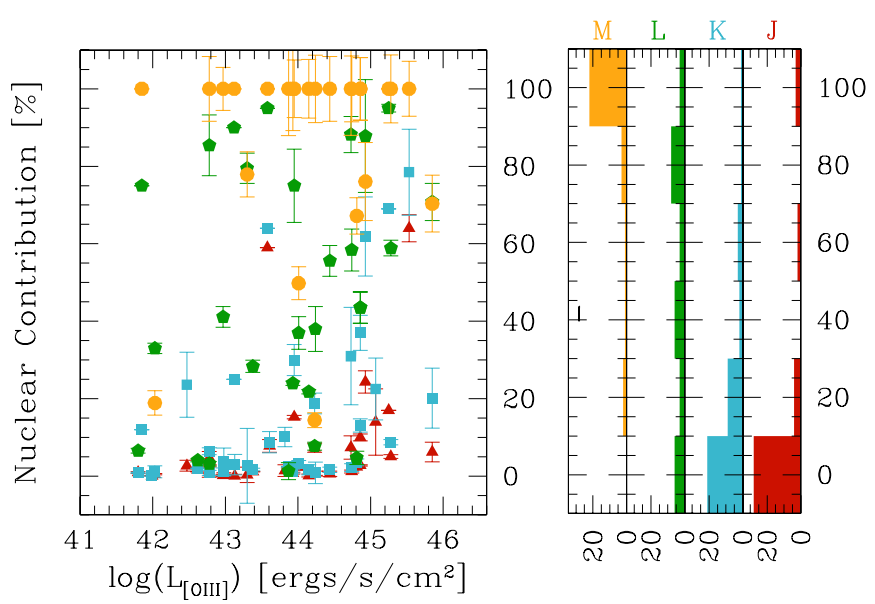

Figure 4. Contribution from the nuclear flux to the total flux as a function of band: $J, K, L$, and $M$ measurements are presented with triangles, squares, pentagons, and circles, respectively. The right-hand side histograms show the projected distributions.

(A color version of this figure is available in the online journal.)

Table 6

Mean, Standard Deviation, Skewness, Median, and Mode Percentage Contribution of Each Galactic Component

\begin{tabular}{|c|c|c|c|c|c|c|c|}
\hline & Band & Number & $\begin{array}{c}\text { Mean } \\
(\%)\end{array}$ & $\begin{array}{c}\sigma \\
(\%)\end{array}$ & $\begin{array}{c}\text { Skew } \\
(\%)\end{array}$ & $\begin{array}{c}\text { Median } \\
(\%)\end{array}$ & $\begin{array}{c}\text { Mode } \\
(\%)\end{array}$ \\
\hline \multirow[t]{5}{*}{ Nucleus } & $M$ & $20 / 28$ & 84 & 27 & -1.5 & 100 & 100 \\
\hline & $L$ & $25 / 28$ & 42 & 28 & 0.2 & 38 & 44 \\
\hline & $K$ & $32 / 34$ & 13 & 18 & 2.1 & 3.3 & 13 \\
\hline & $H$ & $31 / 34$ & 8.4 & 14 & 2.4 & 1.8 & 0.8 \\
\hline & $J$ & $32 / 34$ & 5.9 & 12 & 3.9 & 1.6 & 0.8 \\
\hline \multirow[t]{5}{*}{ Bulge } & $M$ & $7 / 28$ & 44 & 31 & 0.3 & 30 & 44 \\
\hline & $L$ & $25 / 28$ & 52 & 28 & 0.1 & 53 & 57 \\
\hline & $K$ & $34 / 34$ & 35 & 25 & 0.9 & 29 & 35 \\
\hline & $H$ & $34 / 34$ & 33 & 22 & 1.0 & 27 & 33 \\
\hline & $J$ & $34 / 34$ & 34 & 23 & 0.9 & 27 & 34 \\
\hline \multirow[t]{5}{*}{ Disk } & $M$ & $0 / 28$ & $\ldots$ & $\ldots$ & $\ldots$ & $\ldots$ & $\ldots$ \\
\hline & $L$ & $4 / 28$ & 36 & 21 & -0.2 & 37 & 36 \\
\hline & $K$ & $31 / 34$ & 47 & 25 & 0.1 & 42 & 47 \\
\hline & $H$ & $31 / 34$ & 52 & 25 & -0.2 & 51 & 52 \\
\hline & $J$ & $31 / 34$ & 55 & 24 & -0.1 & 48 & 55 \\
\hline \multirow[t]{5}{*}{ Bar } & $M$ & $0 / 28$ & $\ldots$ & $\ldots$ & $\ldots$ & $\ldots$ & $\ldots$ \\
\hline & $L$ & $1 / 28$ & 17 & $\ldots$ & $\ldots$ & 17 & 17 \\
\hline & $K$ & $12 / 34$ & 28 & 18 & 0.5 & 26 & 28 \\
\hline & $H$ & $12 / 34$ & 30 & 17 & -0.3 & 31 & 30 \\
\hline & $J$ & $12 / 34$ & 30 & 17 & 0.1 & 31 & 30 \\
\hline
\end{tabular}

Notes. In column "Number" we indicate the number of fits which include that particular component over the total number of images available in a particular band. Results obtained by AH03 are not included in this table.

the O III luminosities (tabulated in Paper II). It can be seen that, as expected, the percentage nuclear contribution increases for more luminous AGNs, as indicated by the O III measurements (Kotilainen et al. 1992; Alonso-Herrero et al. 2001, 2003).

The fitted parameters for the galactic structures are tabulated in Table 7. In the Appendix details of the individual SBP fittings are given. The fits for the MIR profiles of all the galaxies are shown at two different scales: a detailed one (bottom panels), showing the central part of the galaxy, and a global one (middle panels), where the whole galaxy is observable.

\subsection{Spectral Energy Distributions}

The constructed nuclear IR SEDs are shown in Figure 5. The nuclear fluxes and standard deviations for each observed band 
Table 7

Fitted Parameters of the Galaxy Deconvolution in Each Band of Observation

\begin{tabular}{|c|c|c|c|c|c|c|c|c|c|c|}
\hline Galaxy & Band & $\left(\mathrm{W} \mathrm{m}^{-2} \mu^{-1} \operatorname{arcsec}^{-2}\right)$ & $\left(\mathrm{W} \mathrm{m}^{-2} \mu^{\sigma_{\mathrm{Bul}}} \operatorname{arcsec}^{-2}\right)$ & $\begin{array}{l}r_{\mathrm{eff}}^{\mathrm{Bul}} \\
(\mathrm{pc})\end{array}$ & $n_{\mathrm{Bul}}$ & $\left(\mathrm{W} \mathrm{m}^{-2} \mu^{-1} \operatorname{arcsec}_{\mathrm{dis}}{ }^{-2}\right)$ & $\begin{array}{l}r_{\mathrm{dis}} \\
(\mathrm{pc})\end{array}$ & $\left(\mathrm{W} \mathrm{m}^{-2} \mu^{-1} \operatorname{arcsec}_{\mathrm{bar}}^{-2}\right)$ & $\begin{array}{l}r_{\mathrm{eff}}^{\mathrm{bar}} \\
(\mathrm{pc})\end{array}$ & $n_{\text {bar }}$ \\
\hline \multirow[t]{5}{*}{ NGC 34} & $J$ & $5.6 e-14$ & $2.4 \mathrm{e}-14$ & 269 & 1.4 & $1.2 \mathrm{e}-15$ & 1102 & $\ldots$ & $\ldots$ & $\ldots$ \\
\hline & $H$ & $1.2 \mathrm{e}-13$ & $2.9 \mathrm{e}-14$ & 225 & 1.3 & $1.5 \mathrm{e}-15$ & 1071 & $\ldots$ & $\ldots$ & $\ldots$ \\
\hline & $K$ & $4.8 \mathrm{e}-14$ & $3.1 \mathrm{e}-14$ & 292 & 2.2 & $3.3 e-16$ & 1134 & $\ldots$ & $\ldots$ & $\ldots$ \\
\hline & $L$ & $2.9 \mathrm{e}-14$ & $1.0 \mathrm{e}-14$ & 162 & 1.2 & $9.7 e-17$ & 980 & $\ldots$ & $\ldots$ & $\ldots$ \\
\hline & $M$ & $1.5 e-14$ & $7.5 e-15$ & 225 & 1.8 & $\ldots$ & $\ldots$ & $\ldots$ & $\ldots$ & $\ldots$ \\
\hline \multirow[t]{5}{*}{ IRAS $00198-7926 \mathrm{~N}$} & $J$ & $9.0 \mathrm{e}-15$ & $2.9 \mathrm{e}-16$ & 1397 & 0.5 & $8.8 \mathrm{e}-17$ & 7765 & $\ldots$ & $\ldots$ & $\ldots$ \\
\hline & $H$ & $6.7 \mathrm{e}-15$ & $2.3 e-16$ & 1722 & 0.5 & $5.0 \mathrm{e}-17$ & 8908 & $\ldots$ & $\ldots$ & $\ldots$ \\
\hline & $K$ & $5.7 e-15$ & $9.5 e-17$ & 1559 & 0.3 & $3.5 e-17$ & 8670 & $\ldots$ & $\ldots$ & $\ldots$ \\
\hline & $L$ & $6.6 e-14$ & $6.3 e-16$ & 1262 & 0.5 & $\ldots$ & $\ldots$ & $\ldots$ & $\ldots$ & $\ldots$ \\
\hline & $M$ & $9.5 e-14$ & $\ldots$ & $\ldots$ & $\ldots$ & $\ldots$ & $\ldots$ & $\ldots$ & $\ldots$ & $\ldots$ \\
\hline \multirow[t]{5}{*}{ IRA $00198-7926 \mathrm{~S}$} & $J$ & $3.4 \mathrm{e}-15$ & $5.3 e-16$ & 2450 & 0.9 & $1.9 \mathrm{e}-16$ & 5939 & $\ldots$ & $\ldots$ & $\ldots$ \\
\hline & $H$ & $8.2 \mathrm{e}-15$ & $2.4 \mathrm{e}-16$ & 2108 & 0.5 & $1.5 \mathrm{e}-16$ & 5671 & $\ldots$ & $\ldots$ & $\ldots$ \\
\hline & $K$ & $2.8 \mathrm{e}-14$ & $6.6 e-17$ & 2197 & 0.2 & $1.0 \mathrm{e}-16$ & 5538 & $\ldots$ & $\ldots$ & $\ldots$ \\
\hline & $L$ & $6.6 \mathrm{e}-14$ & $6.3 e-16$ & 1262 & 0.5 & $\ldots$ & $\ldots$ & $\ldots$ & $\ldots$ & $\ldots$ \\
\hline & $M$ & $9.5 e-14$ & $\ldots$ & $\ldots$ & $\ldots$ & $\ldots$ & $\ldots$ & $\ldots$ & $\ldots$ & $\ldots$ \\
\hline \multirow[t]{4}{*}{ IRAS 00521-7054 } & $J$ & $5.7 e-14$ & $5.0 \mathrm{e}-16$ & 4225 & 1.4 & $\ldots$ & $\ldots$ & $\ldots$ & $\ldots$ & $\ldots$ \\
\hline & $H$ & $7.5 \mathrm{e}-14$ & $2.4 \mathrm{e}-15$ & 3004 & 1.9 & $\ldots$ & $\ldots$ & $\ldots$ & $\ldots$ & $\ldots$ \\
\hline & $K$ & $1.2 \mathrm{e}-13$ & $4.8 \mathrm{e}-16$ & 2821 & 1.2 & $\ldots$ & $\ldots$ & $\ldots$ & $\ldots$ & $\ldots$ \\
\hline & $L$ & $1.7 e-16$ & $8.0 \mathrm{e}-16$ & 3088 & 1.9 & $\ldots$ & $\ldots$ & $\ldots$ & $\ldots$ & $\ldots$ \\
\hline \multirow[t]{5}{*}{ ESO 541-IG12 } & $J$ & $4.3 e-14$ & $9.9 e-16$ & 2460 & 1.1 & $7.9 \mathrm{e}-17$ & 5763 & $\ldots$ & $\ldots$ & $\ldots$ \\
\hline & $H$ & $9.5 e-14$ & $7.6 e-16$ & 2506 & 1.0 & $4.4 \mathrm{e}-17$ & 6810 & $\ldots$ & $\ldots$ & $\ldots$ \\
\hline & $K$ & $1.3 e-13$ & $8.7 e-16$ & 2346 & 1.4 & $1.9 \mathrm{e}-17$ & 6902 & $\ldots$ & $\ldots$ & $\ldots$ \\
\hline & $L$ & $4.4 \mathrm{e}-13$ & $3.3 e-16$ & 2153 & 1.4 & $\ldots$ & $\ldots$ & $\ldots$ & $\ldots$ & $\ldots$ \\
\hline & $M$ & $2.5 \mathrm{e}-13$ & $3.5 e-16$ & 2107 & 1.4 & $\ldots$ & $\ldots$ & $\ldots$ & $\ldots$ & $\ldots$ \\
\hline \multirow[t]{4}{*}{ IRAS $01475-0740$} & $J$ & $2.2 \mathrm{e}-14$ & $1.3 e-15$ & 466 & 0.9 & $1.3 e-16$ & 1090 & $\ldots$ & $\ldots$ & $\ldots$ \\
\hline & $H$ & $2.2 \mathrm{e}-14$ & $1.4 \mathrm{e}-15$ & 462 & 1.0 & $1.4 \mathrm{e}-16$ & 799 & $\ldots$ & $\ldots$ & $\ldots$ \\
\hline & $K$ & $2.0 \mathrm{e}-14$ & $4.7 e-16$ & 444 & 0.9 & $4.9 e-17$ & 852 & $\ldots$ & $\ldots$ & $\ldots$ \\
\hline & $L$ & $5.9 \mathrm{e}-14$ & $1.6 e-16$ & 380 & 1.0 & $\ldots$ & $\ldots$ & $\ldots$ & $\ldots$ & $\ldots$ \\
\hline \multirow[t]{4}{*}{ NGC 1144} & $J$ & $1.6 e-14$ & $4.1 e-15$ & 404 & 0.6 & $1.6 e-15$ & 2478 & $\ldots$ & $\ldots$ & $\ldots$ \\
\hline & $H$ & $1.1 \mathrm{e}-14$ & $3.6 e-15$ & 422 & 0.7 & $1.0 \mathrm{e}-15$ & 2478 & $\ldots$ & $\ldots$ & $\ldots$ \\
\hline & $K$ & $8.0 \mathrm{e}-15$ & $1.4 \mathrm{e}-15$ & 399 & 0.6 & $4.4 \mathrm{e}-16$ & 2253 & $\ldots$ & $\ldots$ & $\ldots$ \\
\hline & $L$ & $2.1 \mathrm{e}-14$ & $3.7 e-16$ & 329 & 0.9 & $1.0 \mathrm{e}-16$ & 2149 & $\ldots$ & $\ldots$ & $\ldots$ \\
\hline \multirow[t]{4}{*}{$\mathrm{MCG}-2-8-39$} & $J$ & $3.5 e-15$ & $2.1 \mathrm{e}-15$ & 1762 & 1.6 & $2.6 e-17$ & 11483 & $2.4 \mathrm{e}-17$ & 3216 & 0.2 \\
\hline & $H$ & $2.1 \mathrm{e}-15$ & $1.5 e-17$ & 2698 & 0.2 & $1.2 \mathrm{e}-17$ & 17104 & $1.9 e-15$ & 1971 & 1.8 \\
\hline & $K$ & $1.5 \mathrm{e}-15$ & $1.4 \mathrm{e}-15$ & 2076 & 2.1 & $7.5 e-18$ & 14443 & $7.4 \mathrm{e}-18$ & 2550 & 0.2 \\
\hline & $L$ & $1.2 \mathrm{e}-14$ & $2.9 \mathrm{e}-16$ & 1750 & 2.0 & $\ldots$ & $\ldots$ & $\ldots$ & $\ldots$ & $\ldots$ \\
\hline \multirow[t]{3}{*}{ NGC 1194} & $J$ & $1.1 \mathrm{e}-15$ & $2.5 \mathrm{e}-14$ & 3877 & 3.3 & $2.5 \mathrm{e}-17$ & 7256 & $\ldots$ & $\ldots$ & $\ldots$ \\
\hline & $H$ & $4.7 e-15$ & $2.3 e-14$ & 3288 & 3.4 & $3.7 e-17$ & 4109 & $\ldots$ & $\ldots$ & $\ldots$ \\
\hline & $K$ & $1.8 \mathrm{e}-14$ & $1.3 e-14$ & 3313 & 3.5 & $1.1 \mathrm{e}-17$ & 5935 & $\ldots$ & $\ldots$ & $\ldots$ \\
\hline \multirow[t]{3}{*}{ NGC 1320} & $J$ & $7.3 e-15$ & $7.7 e-15$ & 1597 & 2.3 & $1.2 \mathrm{e}-16$ & 3091 & $\ldots$ & $\ldots$ & $\ldots$ \\
\hline & $H$ & $1.7 e-14$ & $5.8 \mathrm{e}-15$ & 1357 & 2.1 & $1.0 \mathrm{e}-16$ & 3238 & $\ldots$ & $\ldots$ & $\ldots$ \\
\hline & $K$ & $2.6 e-14$ & $2.6 e-15$ & 1818 & 2.3 & $3.2 \mathrm{e}-17$ & 3203 & $\ldots$ & $\ldots$ & $\ldots$ \\
\hline
\end{tabular}

(This table is available in its entirety in a machine-readable form in the online journal. A portion is shown here for guidance regarding its form and content.)

are summarized in Table 8. Here we include the SEDs already determined by Alonso-Herrero et al. (2001, 2003). Upper limits when the galaxy was undetected in $L, M$, and $N$ bands are also shown.

There are some cases where a lack of photometric points prevents us from obtaining a good SED, as with NGC 1194, IRAS 03362-1642, ESO 33-G2, NGC 4941, and NGC 7590. The SEDs of both nuclei in IRAS 00198-7926 are similar and do not determine whether the northern or southern nucleus (or both) corresponds to the AGN.

Visual inspection of the SEDs reveals that we can broadly group most of them into two types depending on the NIR behavior: one group shows a "classic" bell-shaped SED, as expected from the emission of a dusty torus, while the remaining SEDs show a much flatter NIR spectral distribution, with a clear excess with respect to the first group. We have quantified this behavior by measuring the NIR slope of the SEDs and classify them depending on whether the slope $\alpha\left(\lambda f_{\lambda} \propto \lambda^{\alpha}\right)$ is larger or smaller than 1. Individual and average SEDs for these two groups can be seen in Figure 6. The tabulated values for the NIR slope are shown in Table 8. Note that some values have rather large errors and therefore the classification can be ambiguous. The possible nature of the SEDs presenting a NIR excess is discussed in the next section and further analysis can be found in Paper II.

In the same figure we also include the SEDs of the three $\mathrm{H}$ II nuclei found in our sample and of NGC 34 and NGC 5953 where contamination by a starburst component is likely. It can be seen that the morphology of their SEDs clearly departs from the bell-shaped spectra of the first group of Seyfert nuclei and are somewhat closer to the second group of Seyfert galaxies, as it is also noted by the flat value of their NIR slopes (see Table 8). 

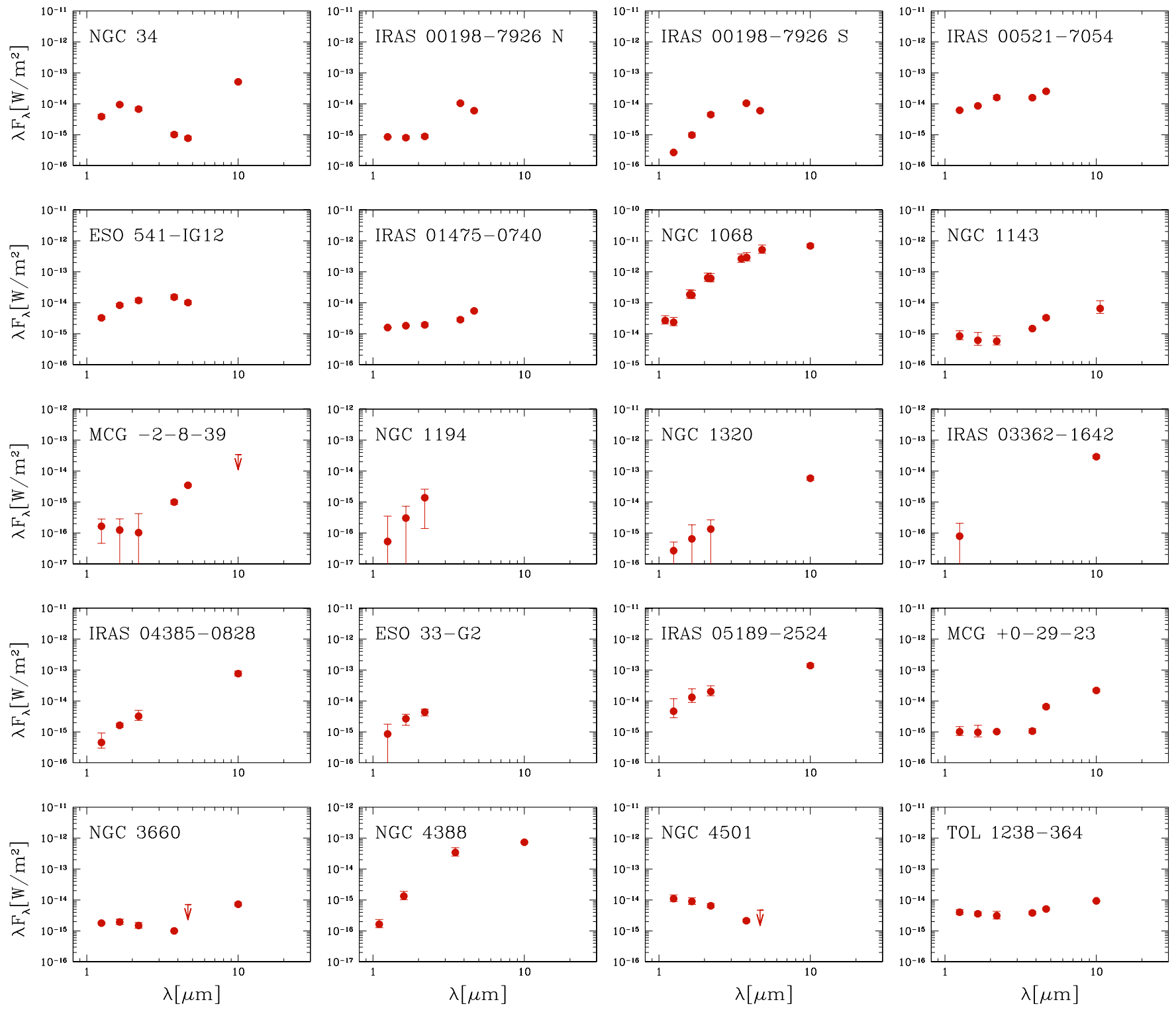

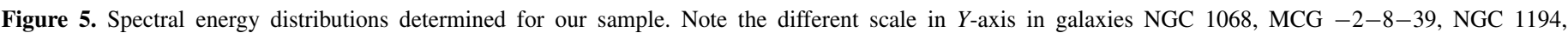
IRAS 03362-1642, NGC 5506, and NGC 7590. The six SEDs are from Alonso-Herrero et al. (2003).

(A color version of this figure is available in the online journal.)

\subsection{Near-IR Excess}

In a Type I Seyfert, where the inner region of the central source is directly observed, the accretion disk can make a significant contribution to the total near-IR emission. Recently, Landt et al. (2011) and Lira et al. (2011) have shown that this is necessary to explain the continuum around $1 \mu \mathrm{m}$ in these objects.

A "typical" Type II source, on the other hand, should have an SED that decreases monotonically toward shorter wavelengths. However, inspection of the SEDs in this work shows several NIR SEDs with a clear excess which resembles Type I systems (see Figure 5 and Table 9).

All galaxies in our sample had an early classification as Type II systems (with MCG -3-34-64 being classified as Seyfert 1.8, NGC 5506 as a Seyfert 1.9, and NGC 7130 as a LINER). However, as noted above, more recent observations show that NGC 6810, MRK 897, and MCG +0-29-23 correspond to H II nuclei, while NGC 34 and NGC 5953 might have contamination by a starburst component. An IR excess is expected for these objects because, in the absence of an active nucleus, the Rayleigh-Jeans emission tail from cold stars dominates this spectral region for moderately old starbursts $(\sim$ a few dozen Myr). This is clearly observed in other starbursts and extensively modeled (e.g., Efstathiou et al. 2000; Dopita et al. 2005; Rodríguez-Merino et al. 2011).

The remaining Seyfert II objects that show an IR excess require alternative explanations; we discuss various mechanisms below.

A traditional torus - a spatially continuous structure with large optical depth-would completely obscure the accretion disk in Type II objects, but a clumpy torus gives a non-zero probability of observing the central source at any orientation; the latter scenario is increasingly accepted and could explain some near-IR emission.

Alternatively, a nuclear jet could make a significant contribution to the observed near-IR fluxes. In this case the emission would be due to synchrotron instead of thermal processes. Jet emission is found to be prominent in radio-loud AGNs and in relativistically boosted radio-quiet AGNs (also known as radiointermediate AGNs; Falcke et al. 1996; Barvainis et al. 2005). 

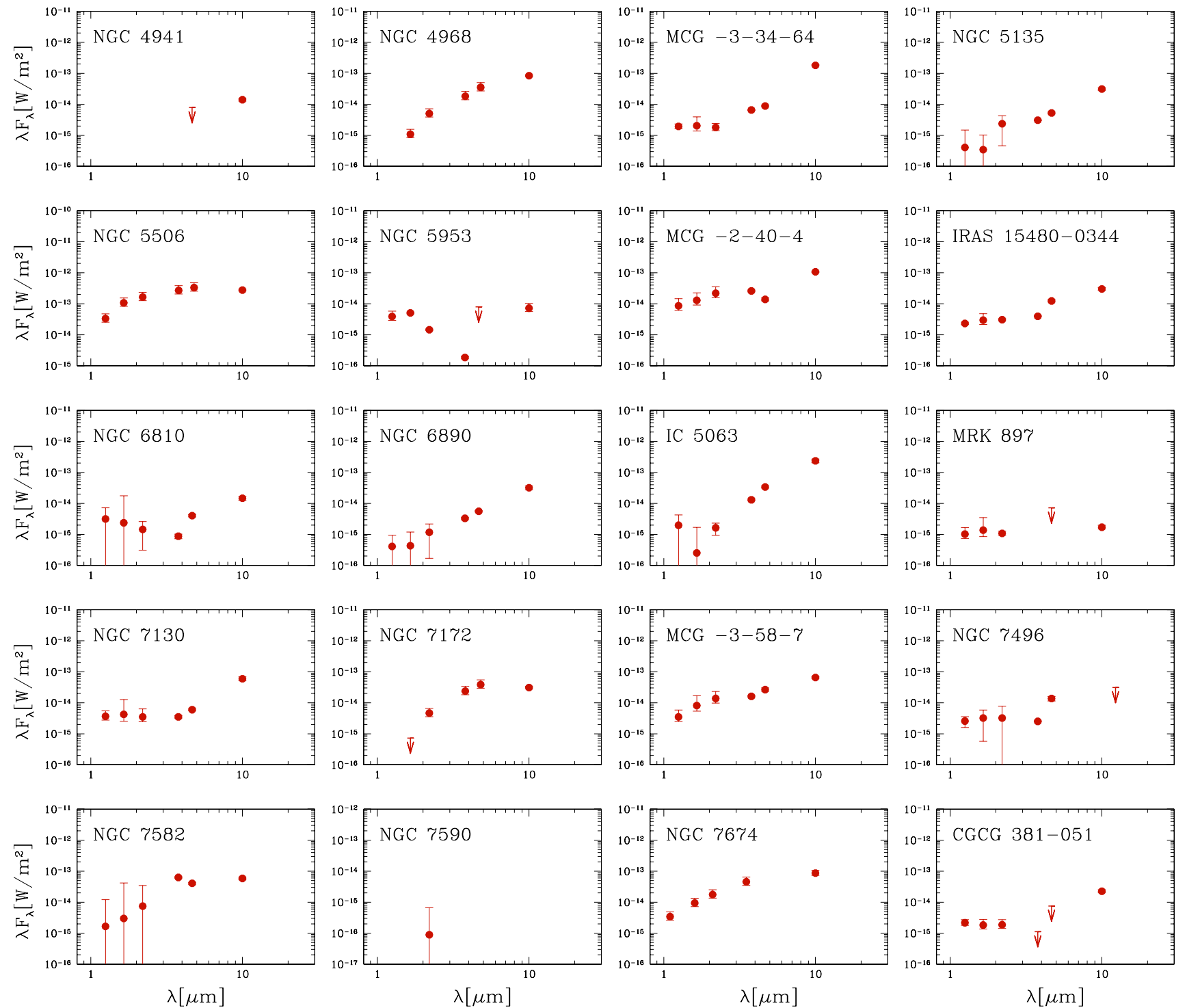

Figure 5. (Continued)
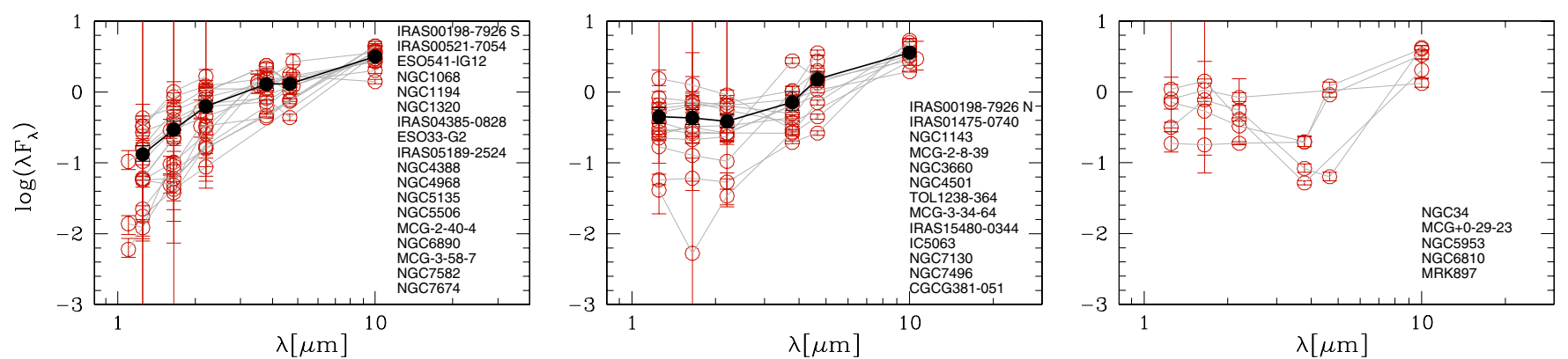

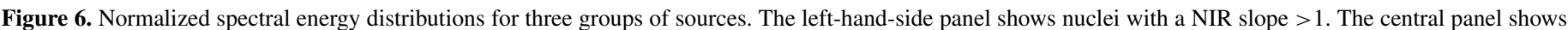

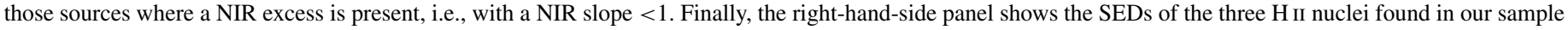

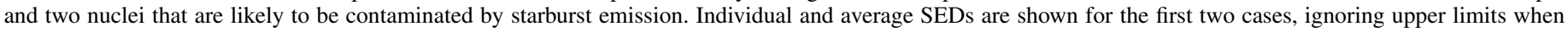
deriving the averages.

(A color version of this figure is available in the online journal.)

Radio-loud AGNs are defined as those with $R_{L}>100$ (where $R_{L}=F_{5 \mathrm{GHz}} / F_{B}$; Kellermann et al. 1989), radio-intermediate AGNs are those with $3-10 \lesssim R_{L} \lesssim 100$, while radio-quiet AGNs have $R_{L} \ll 10$.

We have compiled $5 \mathrm{GHz}$ measurements for our sample and determined $R_{L}$ by computing $L^{\text {bol }}$ from measured [O III] fluxes and then turning them into nuclear $B$ fluxes following Marconi et al. (2004). The resulting $R_{L}$ values are presented here for the SEDs with NIR excess and in Paper II for the complete sample. We found that $\sim 30 \%$ of the sources with NIR excess can be classified as radio-loud (F 01475-0740, NGC 4501, and perhaps NGC 7496); only two additional radio-loud sources 
Table 8

Nuclear Fluxes

\begin{tabular}{|c|c|c|c|c|c|c|c|}
\hline Galaxy & $\begin{array}{c}J \text { Band } \\
(\mathrm{mJy})\end{array}$ & $\begin{array}{c}H \text { Band } \\
(\mathrm{mJy})\end{array}$ & $\begin{array}{c}K \text { Band } \\
(\mathrm{mJy})\end{array}$ & $\begin{array}{c}L \text { Band } \\
(\mathrm{mJy})\end{array}$ & $\begin{array}{c}M \text { Band } \\
(\mathrm{mJy})\end{array}$ & $\begin{array}{c}N \text { Band } \\
(\mathrm{mJy})\end{array}$ & NIR Slope ${ }^{a}$ \\
\hline NGC 34 & $1.60 \pm 0.25$ & $5.17 \pm 0.28$ & $4.92 \pm 0.70$ & $1.27 \pm 0.17$ & $1.19 \pm 0.16$ & $171 \pm 12$ & $0.97 \pm 1.26$ \\
\hline IRAS 00198-7926 N & $0.35 \pm 0.02$ & $0.44 \pm 0.05$ & $0.65 \pm 0.08$ & $13.10 \pm 1.21$ & $9.27 \pm 0.75$ & $\ldots$ & $0.10 \pm 0.15$ \\
\hline IRAS 00198-7926 S & $0.11 \pm 0.01$ & $0.54 \pm 0.08$ & $3.28 \pm 0.43$ & $13.10 \pm 1.21$ & $9.27 \pm 0.75$ & $\ldots$ & $5.02 \pm 0.16$ \\
\hline IRAS 00521-7054 & $2.57 \pm 0.14$ & $4.70 \pm 0.29$ & $11.66 \pm 1.65$ & $19.91 \pm 1.84$ & $39.31 \pm 2.79$ & $\ldots$ & $1.69 \pm 0.28$ \\
\hline ESO 541-IG12 & $1.35 \pm 0.16$ & $4.56 \pm 0.56$ & $8.73 \pm 1.44$ & $19.15 \pm 3.18$ & $15.71 \pm 2.08$ & $<140$ & $2.28 \pm 0.62$ \\
\hline IRAS 01475-0740 & $0.66 \pm 0.02$ & $1.00 \pm 0.04$ & $1.41 \pm 0.19$ & $3.57 \pm 0.45$ & $8.47 \pm 0.63$ & $\ldots$ & $0.34 \pm 0.09$ \\
\hline NGC $1068^{b}$ & 9.8 & 97.6 & 449.6 & 3691 & 8245 & 23000 & $5.77 \pm 0.86$ \\
\hline NGC 1144 & $0.35 \pm 0.11$ & $0.34 \pm 0.15$ & $0.42 \pm 0.14$ & $1.84 \pm 0.04$ & $5.10 \pm 0.55$ & $23 \pm 10^{\mathrm{c}}$ & $-0.67 \pm 0.24$ \\
\hline MCG-2-8-39 & $0.07 \pm 0.05$ & $0.07 \pm 0.09$ & $0.07 \pm 0.23$ & $1.25 \pm 0.19$ & $5.39 \pm 0.47$ & $<114^{\mathrm{c}}$ & $-1.00 \pm 0.01$ \\
\hline NGC 1194 & $0.02 \pm 0.12$ & $0.17 \pm 0.23$ & $1.01 \pm 0.90$ & $\ldots$ & $\ldots$ & $133 \pm 19$ & $5.92 \pm 0.43$ \\
\hline NGC 1320 & $0.11 \pm 0.10$ & $0.35 \pm 0.65$ & $0.98 \pm 0.97$ & $\ldots$ & $\ldots$ & $195 \pm 27$ & $2.86 \pm 0.17$ \\
\hline IRAS 03362-1642 & $0.04 \pm 0.06$ & no-det & no-det & $\ldots$ & $\ldots$ & $970 \pm 110$ & $\ldots$ \\
\hline IRAS 04385-0828 & $0.19 \pm 0.10$ & $0.90 \pm 0.14$ & $2.36 \pm 0.84$ & $\ldots$ & $\ldots$ & $254 \pm 46$ & $3.43 \pm 0.64$ \\
\hline ESO 33-G2 & $0.36 \pm 0.38$ & $1.47 \pm 0.57$ & $3.21 \pm 0.80$ & $\ldots$ & $\ldots$ & $\ldots$ & $2.86 \pm 0.68$ \\
\hline IRAS 05189-2524 & $1.93 \pm 1.18$ & $7.21 \pm 3.37$ & $14.67 \pm 5.23$ & $\ldots$ & $\ldots$ & $463 \pm 62$ & $2.57 \pm 0.66$ \\
\hline $\mathrm{MCG}+0-29-23$ & $0.42 \pm 0.13$ & $0.53 \pm 0.22$ & $0.75 \pm 0.04$ & $1.33 \pm 0.22$ & $10.13 \pm 1.22$ & $73 \pm 7$ & $0.02 \pm 0.11$ \\
\hline NGC 3660 & $0.74 \pm 0.05$ & $1.07 \pm 0.21$ & $1.09 \pm 0.23$ & $1.26 \pm 0.07$ & $<11.07$ & $24.2 \pm 3.3$ & $-0.32 \pm 0.37$ \\
\hline NGC $4388^{b}$ & 0.06 & 0.71 & $\ldots$ & 39.9 & $\ldots$ & $245 \pm 15$ & $4.18 \pm 2.17$ \\
\hline $\mathrm{NGC} 4501^{\mathrm{d}}$ & $4.58 \pm 1.12$ & $4.93 \pm 1.18$ & $4.76 \pm 0.71$ & $2.68 \pm 0.25$ & $<7.22$ & $6.0 \pm 0.5^{\mathrm{c}}$ & $-0.93 \pm 0.11$ \\
\hline TOL 1238-364 & $1.67 \pm 0.30$ & $1.96 \pm 0.27$ & $2.27 \pm 0.64$ & $4.81 \pm 0.55$ & $7.96 \pm 0.68$ & $31.2 \pm 0.4$ & $-0.46 \pm 0.02$ \\
\hline NGC 4941 & no-det & no-det & no-det & $\ldots$ & $<12.23$ & $47 \pm 5$ & $\ldots$ \\
\hline NGC $4968^{b}$ & no-det & 0.6 & 3.7 & 23.2 & 56.5 & $280 \pm 20$ & $5.32 \pm 0.01$ \\
\hline MCG-3-34-64 & $0.81 \pm 0.14$ & $1.13 \pm 0.54$ & $1.32 \pm 0.34$ & $8.29 \pm 0.34$ & $13.75 \pm 0.96$ & $603 \pm 30$ & $-0.14 \pm 0.19$ \\
\hline NGC 5135 & $0.17 \pm 0.45$ & $0.19 \pm 0.38$ & $1.74 \pm 1.41$ & $3.88 \pm 0.10$ & $8.18 \pm 0.62$ & $104 \pm 10$ & $3.16 \pm 2.08$ \\
\hline NGC $5506^{\mathrm{b}}$ & 13.8 & 59.0 & 120.4 & 340.1 & 530.0 & $100 \pm 10$ & $2.83 \pm 0.80$ \\
\hline NGC $5953^{d}$ & $1.61 \pm 0.53$ & $2.77 \pm 0.18$ & $1.06 \pm 0.05$ & $0.23 \pm 0.02$ & $<12.23$ & $24 \pm 7$ & $-1.68 \pm 1.53$ \\
\hline MCG-2-40-4 & $3.57 \pm 1.47$ & $7.10 \pm 3.00$ & $15.99 \pm 6.15$ & $32.63 \pm 2.21$ & $21.43 \pm 2.24$ & $355 \pm 21$ & $1.65 \pm 0.10$ \\
\hline IRAS 15480-0344 & $0.96 \pm 0.09$ & $1.62 \pm 0.62$ & $2.24 \pm 0.18$ & $4.95 \pm 0.17$ & $19.11 \pm 1.68$ & $100 \pm 10$ & $0.50 \pm 0.23$ \\
\hline NGC 6810 & $1.31 \pm 1.70$ & $1.30 \pm 8.41$ & $1.06 \pm 0.84$ & $1.10 \pm 0.18$ & $6.23 \pm 0.52$ & $49 \pm 6.5$ & $-1.37 \pm 0.21$ \\
\hline NGC 6890 & $0.17 \pm 0.22$ & $0.24 \pm 0.42$ & $0.86 \pm 0.73$ & $4.16 \pm 0.27$ & $8.61 \pm 0.48$ & $107 \pm 14$ & $1.89 \pm 0.92$ \\
\hline IC 5063 & $0.82 \pm 0.95$ & $0.14 \pm 0.79$ & $1.20 \pm 0.50$ & $16.38 \pm 1.17$ & $52.48 \pm 4.39$ & $787 \pm 105$ & $-0.37 \pm 4.00$ \\
\hline MRK 897 & $0.43 \pm 0.16$ & $0.75 \pm 0.45$ & $0.79 \pm 0.11$ & & $<11.15$ & $5.7 \pm 0.8$ & $0.08 \pm 0.54$ \\
\hline NGC 7130 & $1.53 \pm 0.50$ & $2.32 \pm 1.56$ & $2.58 \pm 1.15$ & $4.41 \pm 0.41$ & $9.27 \pm 0.79$ & $197 \pm 26$ & $-0.08 \pm 0.33$ \\
\hline NGC $7172^{\mathrm{b}}$ & non-det & $<0.4$ & 3.4 & 30.0 & 61.4 & $103 \pm 10$ & $\ldots$ \\
\hline MCG-3-58-7 & $1.45 \pm 0.59$ & $4.47 \pm 2.31$ & $10.12 \pm 4.11$ & $20.29 \pm 1.07$ & $41.19 \pm 4.94$ & $218 \pm 15$ & $2.42 \pm 0.35$ \\
\hline NGC 7496 & $1.07 \pm 0.40$ & $1.77 \pm 1.44$ & $2.36 \pm 3.31$ & $3.16 \pm 0.13$ & $21.28 \pm 3.55$ & $\ldots$ & $0.39 \pm 0.24$ \\
\hline NGC $7582^{\mathrm{e}}$ & $0.69 \pm 4.33$ & $1.64 \pm 21.24$ & $5.45 \pm 19.84$ & $79.06 \pm 3.88$ & $63.19 \pm 4.72$ & 195 & $2.66 \pm 0.31$ \\
\hline NGC 7590 & no-det & no-det & $0.26 \pm 0.55$ & no-det & $\ldots$ & $\ldots$ & $\ldots$ \\
\hline NGC $7674^{\mathrm{b}}$ & 1.25 & 5.0 & 12.3 & 53 & $\ldots$ & 344 & $3.04 \pm 0.54$ \\
\hline CGCG 381-051 & $0.91 \pm 0.19$ & $1.01 \pm 0.34$ & $1.37 \pm 0.43$ & $<1.41$ & $<11.65$ & $75 \pm 8$ & $-0.27 \pm 0.20$ \\
\hline
\end{tabular}

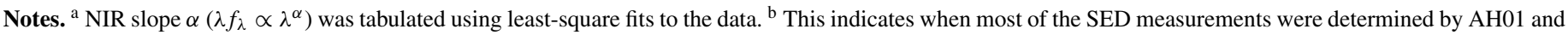

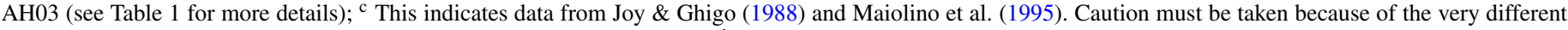

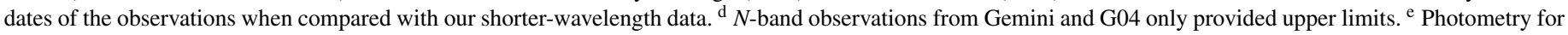
NGC 7582 will be complemented with observations from Prieto et al. (2010) and Ramos-Almeida et al. (2009) in Paper II.

present a bell-shaped SED (F 04385-0828 and NGC 7172), representing a $10 \%$ of that subsample. NGC 4501 presents a rather peculiar SED, with a power-law distribution across the entire $1-10 \mu \mathrm{m}$ range (see Paper II). In this case synchrotron emission might explain the spectral shape.

Thus, jet emission might explain some observed features in the infrared SEDs, but the link is not proven and does not explain all cases.

A very hot dust component has been proposed to explain nearIR emission in Mrk 1239 and Mrk 766 (Rodríguez-Ardila et al. 2005; Rodríguez-Ardila \& Mazzalay 2006). These are Type I AGNs, which provide an unimpaired view of the hottest dust region, and emission from this dust-possibly carbonaceous grains surviving inside the silicate sublimation radius-appears as an excess superimposed on the disk component. Interestingly, the peak observed in these two sources is clearly distinct from the putative torus component (which is not observed in these sources as no data are available beyond $4 \mu \mathrm{m}$ ).

Similarly, a hot dust component was also used in other works when modeling the near-IR region of Spitzer spectra for powerful QSOs (Mor \& Trakhtenbrot 2011; Mor et al. 2009; Schweitzer et al. 2008; Deo et al. 2011). They argue that this component is required to fit the NIR part of the spectrum and that disk emission can only contribute significantly at $1 \mu \mathrm{m}$, but not enough at longer wavelengths. Therefore very hot pure-graphite dust emission is proposed.

A similar very hot dust component could explain some of our observations, but, because of its location inside the torus sublimation radius, it again requires a clumpy absorbing medium to explain its detection.

A very compact nuclear starburst might have a significant contribution to the nuclear fluxes. To test this hypothesis, we 
Table 9

Near-IR Excess and Radio loudness

\begin{tabular}{lccc}
\hline \hline Galaxy & Excess & H II Nuclei & $R_{L}$ \\
\hline IRAS 00198-7926 N & Yes & No & $\ldots$ \\
IRAS 01475-0740 & Yes & No & 70 yes \\
NGC 1144 & Yes & No & 3.4 no \\
MCG -2-8-39 & Yes & No & 0.2 no \\
NGC 3660 & Yes & No & 0.9 no \\
NGC 4501 & Yes & No & 95 yes \\
Tol 1238-364 & Yes & No & 0.4 no \\
MCG -3-34-64 & Yes & No & 2.2 no \\
IRAS 15480-0344 & Yes & No & 0.6 no \\
NGC 7130 & Yes & No & 1.1 no \\
NGC 7496 & Yes & No & 32 yes \\
CGGC 381-051 & Yes & No & 2.4 no \\
\hline
\end{tabular}

have looked at the positions of Seyfert nuclei in the diagnostic diagrams presented in Figure 1, where nuclei with a NIR excess are shown with a square. It can be seen that there is no clear segregation of these nuclei toward the H II location in the plot. Also, these nuclei do not show preference for the presence of a very young stellar continuum as shown in Table 2 .

Finally, a young and luminous nuclear stellar cluster could contribute to the observed near-IR emission. However, with an absolute magnitude $z_{\mathrm{AB}}=-13$ for the most luminous examples (Côté et al. 2006) their flux is not sufficient to make a significant contribution to the observed SEDs.

\section{SUMMARY}

We have constructed nuclear IR SEDs of 40 Type II Seyfert galaxies from ground-based, high-resolution observations. The images were collected between 2003 and 2004 to reduce variability distortion of the SEDs as much as possible and under good seeing conditions.

We also obtained optical spectroscopy for most of the sample. The spectra were modeled using the STARLIGHT code to characterize their stellar continuum properties and emission lines were used to classify the spectra as active or H II nuclei. Three objects from our sample most likely harbor a starburst nucleus and not an AGN, in agreement with other findings.

The surface brightness profiles of each galaxy, in each observed band, were constructed by fitting elliptical isophotes to the images. The profiles were then modeled using a nucleus, bulge, disk and, where necessary, a bar. This procedure was tested using synthetic data and IR observations of the Andromeda galaxy, which showed that the scale length of the different galactic structures does not change when observing at longer wavelengths. This allowed us to fix the scale lengths in the MIR when analyzing undetected components of distant galaxies. Careful modeling of the PSF was also included to take into account seeing distortions.

The fitted SBP parameters for any particular object are generally consistent between bands. In some cases, as in IRAS 03362-1642, NGC 4941, and NGC 7590, no nuclear component was detected by the deconvolution process in the NIR.

A variety of SED shapes is found in our sample, despite the common Type II Seyfert classification. About $40 \%$ of the Seyfert SEDs are characterized by an upturn or excess in the near-IR. The five objects with strong starburst emission (three $\mathrm{H}$ II nuclei and two Seyfert nuclei with strong contamination from nearby starburst regions) also show a similar excess. For genuine AGNs this component could be explained as emission from a jet, the accretion disk, or from a very hot dust component, leaking out from the central region through a clumpy obscuring structure. The presence of a very compact nuclear starburst as the origin for this NIR excess emission is not favored by our data.

We are forever indebted to Roberto Cid Fernandes for his help using the STARLIGHT code. L.V. gratefully acknowledges fellowship support by project MECESUP UCH0118, and partial support from Fondecyt project 1080603. P.L. is grateful for financial support from Fondecyt grant No. 1080603. This publication was also financed by the ALMA-Conicyt Fund, allocated to project No. 31060003 . A.A.H. acknowledges support from the Spanish Plan Nacional de Astronomía y Astrofísica under grant AYA2009-05705-E. Finally, P.L. expresses all her gratitude to A. Cooke for his never-ending support.

\section{APPENDIX}

\section{NOTES ON THE SURFACE BRIGHTNESS PROFILE DECONVOLUTION OF INDIVIDUAL SOURCES}

In the notes below, whenever a parameter is described as "constrained" by some other value, it means that the parameter was allowed to vary within a limited range around the constraining value(s). Without this approach, the parameter would adopt a solution that was non-physical or at odds with values found at other wavelengths.

$N G C$ 34. In this object it is clear how the disk becomes weaker compared to the bulge and nucleus from the $J$ to $L$ bands. The disk in the $L$ band had to be constrained to have a similar scale length to the $J, H$, and $K$ bands. In the $M$ band the disk is not visible, but the bulge is still dominant. Here the bulge effective radius had to be restricted to have similar values to those derived at other wavelengths. The fits are shown in Figure 7.

IRAS 00198-7926. The NIR images for this galaxy show two very close compact objects. It is therefore very difficult to determine which of the two corresponds to the active nucleus. We label them as $\mathrm{N}$ and S. Elliptical photometry was performed on both objects in each band. The fitting results are shown in Figure 7 (online). The southern object has a strange behavior, where the effective radius and the exponent of the Sersic's law of the bulge continuously decreases from $J$ to $K$. Only one object is detected in the $L$-band image, and it is included in both SEDs (north and south) because it is impossible to determine its correspondence with any of the two nuclei. Also, the $L$-band observations seem to suffer from seeing variations, as can be inferred from the fitting results: constraining the parameters of the bulge to be similar to the NIR values produces a very poor fit. As a result, all the emission observed in this band was considered as coming from the nucleus.

IRAS 00521-7054. This galaxy is classified as an E-S0 but, in order to obtain a good fit, the disk component had to be excluded. Because the seeing changed between the observation of the PSF star and the galaxy in the $L$ band only the external parts of the observed profile were considered when fitting the bulge (using the values found in the NIR bands) and the nuclear flux was obtained assuming $F_{\text {nuc }}=F_{\text {gal }}-F_{\text {bul }}$.

ESO 541-IG12. This is a "text-book" active galaxy, with a bright dominant nuclear source. However, in the $L$ band the Sérsic index was constrained by the values obtained in the NIR fits, and in the $M$ band the effective radius of the bulge was constrained to the values obtained in the NIR and $L$ band. 

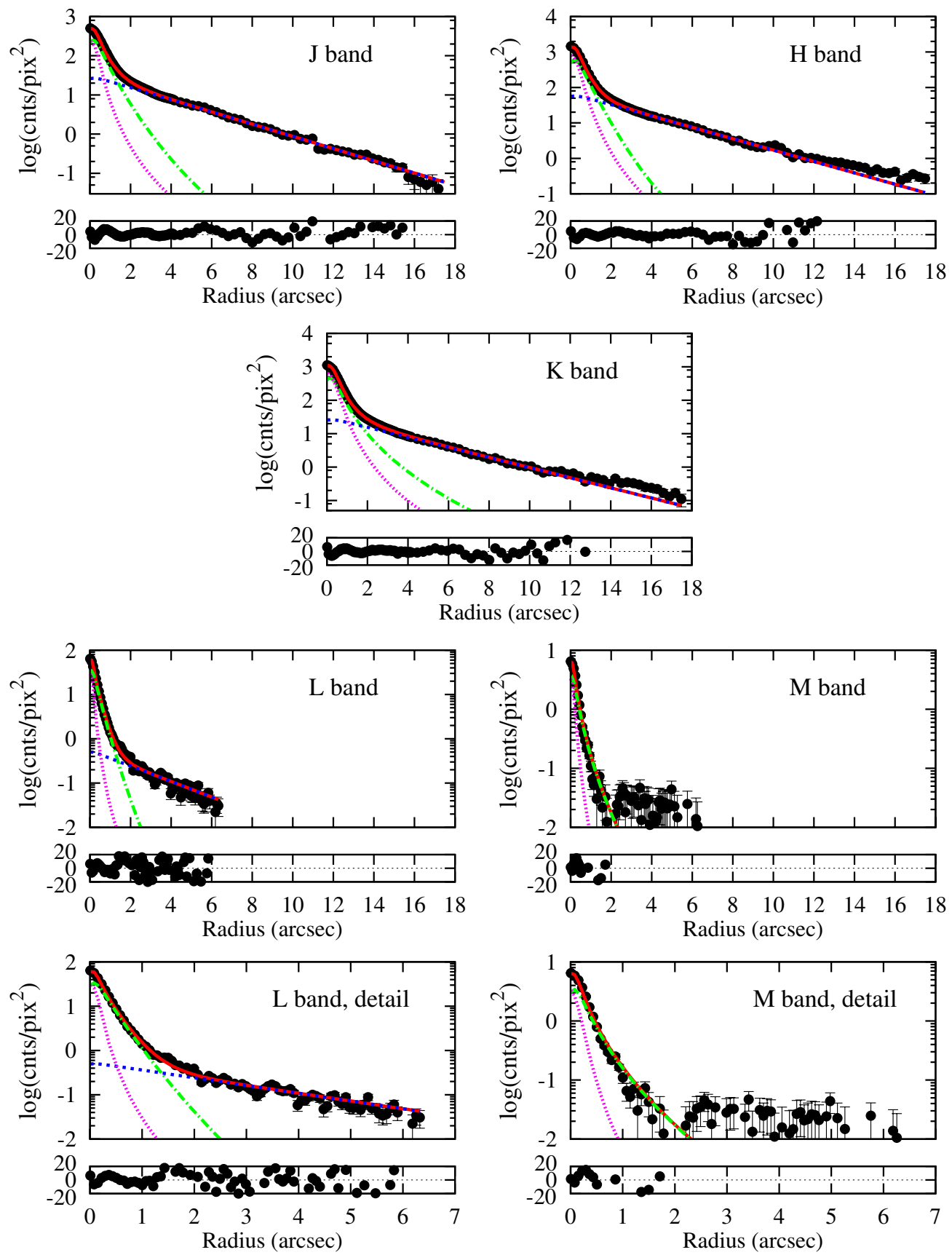

Figure 7. Radial surface brightness profile of the $J-, H-, K-, L-$, and $M$-band observations of NGC 34 (black dots with error bars). The $y$-axis corresponds to log(counts pixel $^{-2}$ ) and the ordinates show the distance from the center in arcseconds. The best-fit model is shown with a thick continuous line. Residuals in percentage are shown in the bottom part of each panel. The different physical components of the model are the nucleus (dotted line), the bulge (dashed-dotted line), the disk (dashed line), and a bar whenever necessary (dashed-double-dotted line). The mid-IR profiles ( $L, M$, and $N$, if available) are plotted in two scales: a detailed scale, showing the central part of the galaxy (bottom panels), and a global scale, showing the complete galaxy (middle panels). Color plots for this and the remaining galaxies are available in the online version of the journal.

(The complete figure set (34 images) and a color version of this figure are available in the online journal.)

$N G C$ 424. The NIR observations could not be flux calibrated and the MIR observations have no PSF star. Hence, no SED was obtained.

IRAS 01475-0740. In order to obtain a reasonable fit to the NIR profiles, the scale length of the disk was constrained to be larger than that of the effective radius of the bulge. In the $L$ band, the size and index of the bulge were restricted to have similar values to those found in the NIR fits.

$N G C$ 1125. This galaxy was only observed in the $L$ and $M$ bands. The disk and bulge parameters could not be restricted and the parameter degeneration was too large. Hence, no SED was obtained.

NGC 1144. This is an interacting system (NGC 1143/44, Arp 118) and the disk of the Seyfert galaxy is very distorted. Comparing the $\mathrm{J}_{-}, \mathrm{H}$-, and $\mathrm{K}$-band images it is evident that the $K$ band is less contaminated by the tidal tails, yielding well behaved parameters for the disk. Using these values to constrain the disk parameters in the $J$ and $H$ bands fitting results are consistent in all three bands. Results are plotted in Figure 7 (online) and clearly show excess emission in the outer parts of 

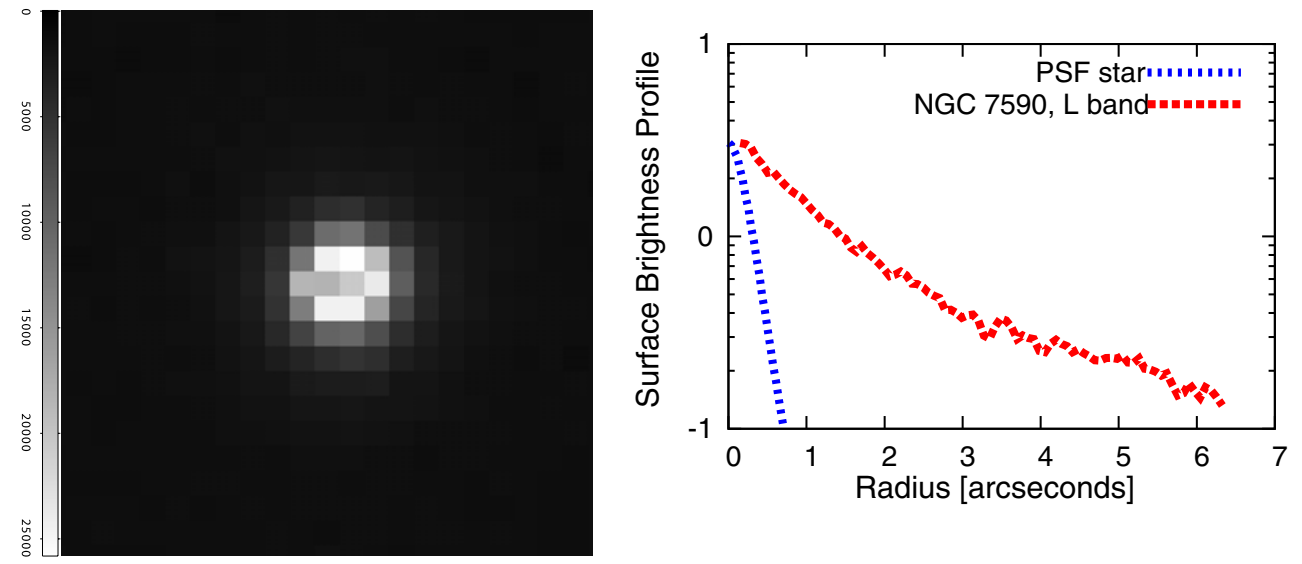

Figure 8. Left: raw image in the $K$ band of galaxy IRAS 05189-2524. The obscured central pixels are evident. Right: comparison of PSF star (dotted-blue line) and galaxy (dashed-red line) radial surface brightness profiles of the $L$ band of galaxy NGC 7590. It is evident that the galaxy is extended unless a strong variation in seeing conditions degraded the galaxy image to a level where no point source can be detected. Adding the fitting results in the near-IR and the resulting Spitzer spectra after star-formation subtraction, it seems this galaxy has at most a very weak AGN.

(A color version of this figure is available in the online journal.)

the galaxy in the $J$ and $H$ bands, coming from the streaming material. In the $L$ band all three components are discernible and the parameters are consistent with those of the NIR once the disk size is constrained to be larger than the bulge size. The galaxy is point-like in the $M$ band, so all the emission is considered as nuclear and no fitting is needed.

$M C G-2-8-39$. This is a very nice example of a barred galaxy. The NIR fits were obtained without any restriction, and the results are consistent. The $L$-band profile was fitted without disk or bar due to the bulge dominating the central region; the size and index of the bulge had to be constrained using the NIR values.

NGC 1194. This galaxy has a highly concentrated bulge $\left(n_{B} \approx 3.3\right)$. The results were obtained constraining the index value of the $K$ band profile to that found in the $J$ and $H$ band fits.

$N G C 1320$. This galaxy was fitted limiting the effective radius of the bulge in the $K$ band.

IRAS 03362-1642. The resulting fit of this galaxy does not include a nuclear component in the $H$ and $K$ bands. In the $J$ band a weak nucleus appears, with a contribution of $\lesssim 1 \%$, almost negligible. The bar is very dominant in all three bands.

IRAS 04385-0828. To fit this galaxy it was necessary to constrain the bulge Sérsic index in the $J$ and $H$ bands to the value found in $K$.

NGC 1667. This galaxy has a very complex morphology, which prevents the determination of a reliable profile fitting in the three bands observed. Hence, no SED was obtained.

$E S O$ 33-G2. No restriction was needed in $J$ and $H$ bands to fit this galaxy. In the $K$ band the disk scale length was constrained to be larger than the bulge and bar sizes, obtaining consistent results for all the parameters.

IRAS 05189-2524. In order to obtain a consistent fit to this galaxy, the disk size in the $H$ band was constrained to be larger than the bulge size. In the $K$ band the first point of the radial profile is lower than the second and third points. This is due to obscuration that can be observed in all the raw images in this band, as shown in the left panel of Figure 8; this first point is not included in the fitting process. In the $N$-band profile, all the emission comes from the nucleus.

ESO 253-G3. This is an interacting system whose two nuclei are too close to fit reliably. Hence, no SED was obtained.

$M C G+0-29-23$. Figure 7 (online) shows the fitting results for this very special object. The results barely change from one band to another and the galaxy is very extended even in the $L$ band. It is not clear whether the central spheroidal component corresponds to a bulge, since the images and the fitted Sérsic index both resembles more closely a bar. In any case, bulge and/or bar, the contribution to the central emission can be determined, even if it is not possible to unveil the exact nature of the component.

$N G C 3660$. This galaxy is very large, and due to the small size of the detector, the galaxy has been truncated in the $J$ and $H$ bands. In order to determine the real value of the disk parameters, the $K$-band image was used and then these fitted values were used to constrain the disk parameters in the $J$ - and $H$-band fits, excluding the profile for $r>45^{\prime \prime}$. In the $L$ band, the profile with $r>2^{\prime \prime}$ was skipped because it is mostly sky contribution. The parameters of the bulge were restricted to those obtained in the $J, H$ and $K$ fits.

$N G C$ 4501. This is a huge galaxy compared to the detector, so the PSF was taken from the standard star. The only restriction needed to obtain consistent results was that the disk size was larger than the bulge size in the $J$ and $H$ bands. In the $L$ band the change in seeing conditions is evident, and to overcome this problem the bulge was fitted for $r>3^{\prime \prime}$, constraining only the Sérsic's index; the nucleus is then computed as $F_{\text {nuc }}=F_{\text {gal }}-F_{\text {bul }}$. The galaxy was not detected in the $M$ or $N$ bands. The upper limit in the $N$ band is that obtained by Gorjian et al. (2004).

TOL 1238-364. The fit to this galaxy in the NIR bands does not include the bump produced by the ending of the flocculent arms between $16^{\prime \prime}$ and $23^{\prime \prime}$. In the $J$ and $H$ bands a disk scale length larger than the bulge size was imposed. In the $K$ band the bulge index was constrained to have similar values to those found in the $J$ and $H$ bands. In the $L$ and $M$ bands the bulge and disk parameters were constrained to values found in the NIR fits. It seems that seeing conditions changed between the observations in the $M$ band, since the PSF star did not produced an acceptable fit for the nucleus, so the bulge was fitted and the difference between the galaxy and the bulge was considered as the nuclear contribution, i.e., $F_{\text {nuc }}=F_{\text {gal }}-F_{\text {bul }}$.

$N G C 4941$. This is a very big galaxy, which means that, again, the PSF is not contained in the galaxy frame but comes from observations of the standard star. Unfortunately, the standard star is not a good approximation to the galaxy PSF, resulting in a null detection of a nucleus in the three NIR band fits. However, 
the galaxy was also not detected in the $L$ and $M$ bands, so it may also be that no detectable near- and MIR emission is associated with the active nucleus.

$M C G-3-34-64$. Without special constraints, the fitted NIR parameters are consistent. In the $L$ and $M$ bands the seeing between the observation of the galaxy and the observation of the PSF star seems to vary, resulting in a poor fit in the $L$ band. In order to fit both profiles, the bulge was fitted with the values found in the NIR fits and the nuclear contribution was calculated as $F_{\text {nuc }}=F_{\text {gal }}-F_{\text {bul }}$.

$N G C 5135$. The bar in this galaxy is very strong and the disk is not seen in the IR images. Between $1^{\prime \prime}$ and $3^{\prime \prime}$ there is a bright star-forming region, dominating the profile at all wavelengths; this region was excluded from the fit in all the profiles. The galaxy profile in the $M$ band looks slightly broader than the PSF star, but the bulge component is not able to account for it, so a change in the seeing conditions is assumed to be responsible. The nuclear flux is calculated from the integration of the galaxy profile for $r \leqslant 1^{\prime \prime}$.

$M R K 463$. This is an interacting system whose two nuclei are too close to fit reliably. Hence, no SED was obtained.

NGC 5953. The bump observed near $r=6^{\prime \prime}$ was excluded because it is produced by the ending of the flocculent arms. In the $L$ band, the seeing is worse in the galaxy observation than the PSF star, as can be seen in Figure 7 (online). In this case, the results of the $J, H$ and $K$ band fits were used to fix the values of the bulge parameters and the flux of the nucleus was estimated as $F_{\text {nuc }}=F_{\text {gal }}-F_{\text {bul }}$.

$M C G-2-40-4$. The bar and the disk are indistinguishable for $r \gtrsim 2^{\prime \prime}$ so the fitted component in the $L$-band is a mixture of both structures. The bulge parameters in this band were constrained to values found in the NIR to reliably separate it from the mixed bar-disk component. The $M$ band profile was fitted with the bulge effective radius and Sérsic's index constrained to the values found in the NIR.

IRAS 15480-0344. Barely no restriction was needed to obtain a good fit in NIR bands. In the $L$ band only a nucleus and a bulge were fitted, and the index of the Sérsic's law was limited by the values found in the NIR bands.

IRAS 19254-7245. This is an interacting system whose two nuclei are too close to fit reliably. Hence, no SED was obtained.

$N G C$ 6810. To obtain a good fit for this galaxy, bumps in the radial profile produced by two large star forming regions were not considered. In the $L, M$ and $N$ bands, the seeing was better in the observations of the PSF star than in the observations of the galaxy. It is impossible for the bulge to account for the difference at $r \lesssim 0$.'5. Fixed values for the bulge parameters were used, similar to the values found at shorter wavelengths, and the nuclear flux was estimated as $F_{\text {nuc }}=F_{\text {gal }}-F_{\text {bul }}$. The extended emission of this galaxy in the $N$ band is an exceptional case.

$N G C$ 6890. To fit this galaxy the bumps at $r \approx 15^{\prime \prime}$ and $r \approx 30^{\prime \prime}$ were excluded from the fitting process because they correspond to the ending of the flocculent arms. In the $L$ band the signal beyond $r \approx 4^{\prime \prime}$ corresponds to sky emission. The galaxy in the $M$ and $N$ bands is consistent with being point-like, so no fit is performed.

IC 5063. This galaxy is classified as SA0, but the best fit uses only bulge and nucleus components (no disk), which get stronger from shorter to longer wavelengths. In the $L$ band we restricted the values of the parameters of the bulge to be close to the values found in the $J, H$, and $K$ fits. In the $M$ band a change in seeing is evident, causing the PSF star to be sharper and narrower than the galaxy. The bulge parameters were frozen and only the nucleus was allowed to vary. The galaxy is point-like in the $N$ band, so no fit is needed.

$M R K 897$. In the $L$ band the object fell too close to the edge of the image and it was not possible to obtain the profile necessary to accurately determine the bulge contribution, so the bulge parameters were restricted to have values similar to the $J, H$, and $K$ ones.

$N G C 7130$. The only restriction needed to fit the NIR bands was that the disk scale length was larger than the bulge effective radius. In the $L$ band the seeing apparently varied between observations of the galaxy and the PSF star. In order to obtain a reliable nuclear flux, the bulge component was fitted between $2^{\prime \prime}$ and $4^{\prime \prime}$ and the parameters constrained to be as close as possible to the $J, H$, and $K$ values. However, the fit to the galaxy was not adequate (mainly in the innermost region), so the nuclear flux is estimated as $F_{\text {nuc }}=F_{\text {gal }}-F_{\text {bul }}$. In the $M$ band, the galaxy looks almost point-like so no fit is needed.

$M C G-3-58-7$. The disk size in the $K$ band image was constrained to be larger than the bulge size. In the $L$ band, only a nucleus and a bulge were included, and the index of the bulge was constrained to have a value similar to those obtained at shorter wavelengths.

$N G C$ 7496. While fitting this galaxy, the $K$ band bulge index had to be restricted by the values obtained in the $J$ and $H$ bands. Also, the disk scale length was restricted to be larger than the bulge effective radius. In the $L$ and $M$ bands only a bulge and a nucleus were included. In $\mathrm{L}$ the bulge size was restricted by the values found in the NIR bands; in M the size and index of the bulge component were constrained by values found at shorter wavelengths.

$N G C$ 7582. The NIR images show a prominent galaxy, orientated nearly face-on, and two very weak arms with a different apparent inclination angle. In the literature, this system has been characterized by an inclination angle of $\sim 60$ degrees (Morris et al. 1985), corresponding to the disk traced by the weak arms. The galaxy has a strong star-forming region near the nucleus, which is also observable in X-rays (Bianchi et al. 2007 ), so the region near $r \approx 2^{\prime \prime} .5$ was not included in the fitting process.

$N G C 7590$. This galaxy was observed twice in the NIR, once in service mode and once in visitor mode, with a different detector each time. The two fits gave consistent results. The point source detected in the NIR bands contributes at most $0.3 \%$ to the total flux. In the $L$ and $M$ bands, no point source was detected, again suggesting a very weak AGN. After subtracting the star forming component (see Paper II) a Spitzer-IRS spectrum of NGC 7590 showed only a weak AGN continuum.

$C G C G$ 381-051. This object was not detected in the $L$ and $M$ bands. In the $J, H$, and $K$ bands the fit did not include the region between $r \approx 15^{\prime \prime}$ and $r \approx 20^{\prime \prime}$, which correspond to the end of the galactic arms. The $H$-band results were used to contains the bulge index in the $K$ band and the bulge size in the $J$ band. Although the bulge is small it can be distinguished from the bar in all images.

\section{REFERENCES}

Allen, M. G., Groves, B. A., Dopita, M. A., Sutherland, R. S., \& Kewley, L. J. 2008, ApJS, 178, 20

Alonso-Herrero, A., Quillen, A. C., Rieke, G. H., Ivanov, V. D., \& Efstathiou, A. 2003, AJ, 126, 81

Alonso-Herrero, A., Quillen, A. C., Simpson, C., Efstathiou, A., \& Ward, M. J. 2001, AJ, 121, 1369

Alonso-Herrero, A., Ward, M. J., \& Kotilainen, J. K. 1996, MNRAS, 278, 902 
Antonucci, R. R. J., \& Miller, J. S. 1985, ApJ, 297, 621

Balcells, M., Graham, A. W., Domínguez-Palmero, L., \& Peletier, R. F. 2003, ApJL, 582, L79

Baldwin, J. A., Phillips, M. M., \& Terlevich, R. 1981, PASP, 93, 5

Barbosa, F. K. B., Storchi-Bergmann, T., Cid Fernandes, R., Winge, C., \& Schmitt, H. 2009, MNRAS, 396, 2

Barmby, P., Ashby, M. L. N., Bianchi, L., et al. 2006, ApJL, 650, L45

Barvainis, R., Lehár, J., Birkinshaw, M., Falcke, H., \& Blundell, K. M. 2005, ApJ, 618, 108

Bassani, L., Dadina, M., Maiolino, R., et al. 1999, ApJS, 121, 473

Baum, S. A., Gallimore, J. F., O’Dea, C. P., et al. 2010, ApJ, 710, 289

Beckert, T., Driebe, T., Hönig, S. F., \& Weigelt, G. 2008, A\&A, 486, L17

Bianchi, S., Chiaberge, M., Piconcelli, E., \& Guainazzi, M. 2007, MNRAS, 374,697

Buchanan, C. L., Gallimore, J. F., O’Dea, C. P., et al. 2006, AJ, 132, 401

Cardelli, J. A., Clayton, G. C., \& Mathis, J. S. 1989, ApJ, 345, 245

Cid Fernandes, R., Gu, Q., Melnick, J., et al. 2004, MNRAS, 355, 273

Cid Fernandes, R., Mateus, A., Sodré, L., Stasińska, G., \& Gomes, J. M. 2005, MNRAS, 358, 363

Côté, P., Piatek, S., Ferrarese, L., et al. 2006, ApJS, 165, 57

Deo, R. P., Richards, G. T., Nikutta, R., et al. 2011, ApJ, 729, 108

Dopita, M. A., Groves, B. A., Fischera, J., et al. 2005, ApJ, 619, 755

Efstathiou, A., Rowan-Robinson, M., \& Siebenmorgen, R. 2000, MNRAS, 313,734

Elvis, M., Risaliti, G., Nicastro, F., et al. 2004, ApJL, 615, L25

Elvis, M., Wilkes, B. J., McDowell, J. C., et al. 1994, ApJS, 95, 1

Falcke, H., Patnaik, A. R., \& Sherwood, W. 1996, ApJL, 473, L13

Falcke, H., Wilson, A. S., \& Simpson, C. 1998, ApJ, 502, 199

Gallimore, J. F., Yzaguirre, A., Jakoboski, J., et al. 2010, ApJS, 187, 172

Gorjian, V., Werner, M. W., Jarrett, T. H., Cole, D. M., \& Ressler, M. E. 2004, ApJ, 605, 156

Graham, A. W. 2001, AJ, 121, 820

Graham, A. W., \& Driver, S. P. 2005, PASA, 22, 118

Grosbøl, P., Patsis, P. A., \& Pompei, E. 2004, A\&A, 423, 849

Hewitt, A., \& Burbidge, G. 1991, ApJS, 75, 297

Huchra, J., \& Burg, R. 1992, ApJ, 393, 90

Hunt, L. K., \& Malkan, M. A. 1999, ApJ, 516, 660

Hunt, L. K., Malkan, M. A., Rush, B., et al. 1999, ApJS, 125, 349

Hunt, L. K., Pierini, D., \& Giovanardi, C. 2004, A\&A, 414, 905

Imanishi, M. 2003, ApJ, 599, 918

Imanishi, M., \& Alonso-Herrero, A. 2004, ApJ, 614, 122

Jaffe, W., Ford, H. C., Ferrarese, L., van den Bosch, F., \& O'Connell, R. W. 1993, Natur, 364, 213

Jaffe, W., Meisenheimer, K., Röttgering, H., Leinert, C., \& Richichi, A. 2004, in IAU Symp. 222, The Interplay Among Black Holes, Stars and ISM in Galactic Nuclei, ed. T. Storchi-Bergmann, L. C. Ho, \& H. R. Schmitt (Cambridge: Cambridge University Press), 37

Joy, M., \& Ghigo, F. D. 1988, ApJ, 332, 179

Kellermann, K. I., Sramek, R., Schmidt, M., Shaffer, D. B., \& Green, R. 1989, AJ, 98, 1195

Kewley, L. J., Heisler, C. A., Dopita, M. A., \& Lumsden, S. 2001, ApJS, 132,37

Kishimoto, M., Hönig, S. F., Antonucci, R., et al. 2011, A\&A, 536, A78

Kishimoto, M., Hönig, S. F., Tristram, K. R. W., \& Weigelt, G. 2009, A\&A, 493, L57

Kormendy, J., \& Kennicutt, R. C., Jr. 2004, ARA\&A, 42, 603

Kotilainen, J. K., Ward, M. J., Boisson, C., Depoy, D. L., \& Smith, M. G. 1992, MNRAS, 256, 149

Landt, H., Elvis, M., Ward, M. J., et al. 2011, MNRAS, 414, 218

Lawrence, A., \& Elvis, M. 1982, ApJ, 256, 410

Lira, P., Arévalo, P., Uttley, P., McHardy, I., \& Breedt, E. 2011, MNRAS, 415,1290

Lira, P., Videla, L., Wu, Y., et al. 2013, ApJ, 764, 159

Maiolino, R., Ruiz, M., Rieke, G. H., \& Keller, L. D. 1995, ApJ, 446, 561

Malkan, M. A., Gorjian, V., \& Tam, R. 1998, ApJS, 117, 25

Marconi, A., Risaliti, G., Gilli, R., et al. 2004, MNRAS, 351, 169

Mass-Hesse, J. M., Rodriguez-Pascual, P. M., Mirabel, I. F., \& Sanz Fernandez de Cordoba, L. 1993, First Light in the Universe. Stars or QSO's? (Gif-surYvette: Editions Frontieres), 397

Meisenheimer, K., Tristram, K. R. W., Jaffe, W., et al. 2007, A\&A, 471,453

Mor, R., Netzer, H., \& Elitzur, M. 2009, ApJ, 705, 298

Mor, R., \& Trakhtenbrot, B. 2011, ApJL, 737, L36
Moran, E. C., Barth, A. J., Kay, L. E., \& Filippenko, A. V. 2000, ApJL, 540, L73

Morris, S., Ward, M., Whittle, M., Wilson, A. S., \& Taylor, K. 1985, MNRAS, 216, 193

Nagar, N. M., \& Wilson, A. S. 1999, ApJ, 516, 97

Nelder, J. A., \& Mead, R. 1965, CompJ, 7, 308

Nenkova, M., Ivezić, Ž., \& Elitzur, M. 2002, ApJL, 570, L9

Nenkova, M., Sirocky, M. M., Ivezić, Ž., \& Elitzur, M. 2008, ApJ, 685, 147

Nenkova, M., Sirocky, M. M., Nikutta, R., Ivezić, Ž., \& Elitzur, M. 2008, ApJ, 685,160

Osterbrock, D. E. 1978, PNAS, 75, 540

Peng, C. Y. 2002, AJ, 124, 294

Penston, M. V., \& Perez, E. 1984, MNRAS, 211, 33P

Pogge, R. W. 1988a, ApJ, 332, 702

Pogge, R. W. 1988b, ApJ, 328, 519

Prieto, M., Aguerri, J. A. L., Varela, A. M., \& Muñoz-Tuñón, C. 2001, A\&A, 367,405

Prieto, M. A., Reunanen, J., \& Kotilainen, J. K. 2002, ApJL, 571, L7

Prieto, M. A., Reunanen, J., Tristram, K. R. W., et al. 2010, MNRAS, 402, 724

Puccetti, S., Fiore, F., Risaliti, G., et al. 2007, MNRAS, 377, 607

Raban, D., Jaffe, W., Röttgering, H., Meisenheimer, K., \& Tristram, K. R. W. 2009, MNRAS, 394, 1325

Rafanelli, P., Osterbrock, D. E., \& Pogge, R. W. 1990, AJ, 99, 53

Ramos Almeida, C., Levenson, N. A., Rodríguez Espinosa, J. M., et al. 2009, ApJ, 702, 1127

Risaliti, G., Maiolino, R., \& Salvati, M. 1999, ApJ, 522, 157

Risaliti, G., Nardini, E., Salvati, M., et al. 2011, MNRAS, 410, 1027

Risaliti, G., Salvati, M., Elvis, M., et al. 2009, MNRAS, 393, L1

Rodríguez-Ardila, A., Contini, M., \& Viegas, S. M. 2005, MNRAS, 357, 220

Rodríguez-Ardila, A., \& Mazzalay, X. 2006, MNRAS, 367, L57

Rodríguez-Merino, L. H., Rosa-González, D., \& Mayya, Y. D. 2011, ApJ, 726,51

Ruiz, M., Rieke, G. H., \& Schmidt, G. D. 1994, ApJ, 423, 608

Rush, B., Malkan, M. A., \& Edelson, R. A. 1996a, ApJ, 473, 130

Rush, B., Malkan, M. A., Fink, H. H., \& Voges, W. 1996b, ApJ, 471, 190

Rush, B., Malkan, M. A., \& Spinoglio, L. 1993, ApJS, 89, 1

Schlegel, D. J., Finkbeiner, D. P., \& Davis, M. 1998, ApJ, 500, 525

Schweitzer, M., Groves, B., Netzer, H., et al. 2008, ApJ, 679, 101

Shi, Y., Rieke, G. H., Hines, D. C., et al. 2006, ApJ, 653, 127

Spinoglio, L., Andreani, P., \& Malkan, M. A. 2002, ApJ, 572, 105

Spinoglio, L., Malkan, M. A., Rush, B., Carrasco, L., \& Recillas-Cruz, E. 1995, ApJ, 453, 616

Strickland, D. K. 2007, MNRAS, 376, 523

Strong, M., Pedlar, A., Aalto, S., et al. 2004, MNRAS, 353, 1151

Swain, M., Vasisht, G., Akeson, R., et al. 2003, ApJL, 596, L163

Thean, A., Pedlar, A., Kukula, M. J., Baum, S. A., \& O'Dea, C. P. 2000, MNRAS, 314,573

Thean, A., Pedlar, A., Kukula, M. J., Baum, S. A., \& O’Dea, C. P. 2001, MNRAS, 325,737

Tommasin, S., Spinoglio, L., Malkan, M. A., \& Fazio, G. 2010, ApJ, 709, 1257

Tommasin, S., Spinoglio, L., Malkan, M. A., et al. 2008, ApJ, 676, 836

Tran, H. D. 2001, ApJL, 554, L19

Tristram, K. R. W., Meisenheimer, K., Jaffe, W., et al. 2007, A\&A, 474, 837

Tristram, K. R. W., Raban, D., Meisenheimer, K., et al. 2009, A\&A, 502, 67

Turner, T. J., George, I. M., Nandra, K., \& Mushotzky, R. F. 1997, ApJ, 488,164

Veilleux, S., \& Osterbrock, D. E. 1987, ApJS, 63, 295

Veilleux, S., Sanders, D. B., \& Kim, D.-C. 1997, ApJ, 484, 92

Veilleux, S., Sanders, D. B., \& Kim, D.-C. 1999, ApJ, 522, 139

Veron-Cetty, M.-P., \& Veron, P. 1991, ESOSR, 10, 1

Weigelt, G., Wittkowski, M., Balega, Y. Y., et al. 2004, A\&A, 425, 77

Weinberger, A. J., Neugebauer, G., \& Matthews, K. 1999, AJ, 117, 2748

Wilson, A. S., \& Tsvetanov, Z. I. 1994, AJ, 107, 1227

Wittkowski, M., Balega, Y., Beckert, T., et al. 1998, A\&A, 329, L45

Wittkowski, M., Kervella, P., Arsenault, R., et al. 2004, A\&A, 418, L39

Wu, Y., Charmandaris, V., Huang, J., Spinoglio, L., \& Tommasin, S. 2009, ApJ, 701,658

Young, S., Hough, J. H., Axon, D. J., Bailey, J. A., \& Ward, M. J. 1995, MNRAS, 272, 513

Young, S., Hough, J. H., Axon, D. J., Ward, M. J., \& Bailey, J. A. 1996, MNRAS, 280, 291

Zitelli, V., Granato, G. L., Mandolesi, N., Wade, R., \& Danese, L. 1993, ApJS, 84,185 